\title{
Gaussian Filters for Nonlinear Filtering Problems
}

\author{
Kazufumi Ito and Kaiqi Xiong *
}

May 25, 1999

\begin{abstract}
In this paper we develop and analyze real-time and accurate filters for nonlinear filtering problems based on the Gaussian distributions. We present the systematic formulation of Gaussian filters and develop efficient and accurate numerical integration of the optimal filter. We also discuss the mixed Gaussian filters in which the conditional probability density is approximated by the sum of Gaussian distributions. A new update rule of weights for Gaussian sum filters is proposed. Our numerical testings demonstrate that new filters significantly improve the extended Kalman filter with no additional cost and the new Gaussian sum filter has a nearly optimal performance.
\end{abstract}

\section{Introduction}

The nonlinear filtering problem consists of estimating the state of a nonlinear stochastic system from noisy observation data. The problem has been the subject of considerable research interest during the past several decades because it has many significant applications in science and engineering such as navigational and guidance systems, radar tracking, sonar ranging and satellite and airplane orbit determination [11, 14, 15]. As is well-known, the most widely used filter is the extended Kalman filter for nonlinear filtering problems. It is derived from the Kalman filter based on the successive linearization of the signal process and the observation map. The extended Kalman filter has been successfully applied to numerous nonlinear filtering problems. If nonlinearities are significant, however, its performance can be substantially improved. Such efforts have also been reported in $[1,5,7,12,13,20]$. In this paper our objective is to develop and analyze real-time and accurate filters for nonlinear filtering algorithms based on Gaussian distributions. We present the systematic formulation of Gaussian filters and mixed Gaussian filters.

We first develop the Gaussian filter. The proposed filter is based on the steps: (1) we assume the conditional probability density to be a Gaussian distribution (i.e., assumed density)

\footnotetext{
${ }^{*}$ Center for Research in Scientific Computation, North Carolina State University, Raleigh, North Carolina 27695-8205. Email: kito@eos.ncsu.edu and xiong@eos.ncsu.edu. This research was in part supported by the Office of Naval Research grant N00014-96-1-0265 and MURI-AFOSR grant F49620-95-1-0447.
} 
and next, (2) we obtain the Gaussian filter by equating the Bayesian formula with respect to the first moment (mean) and the second moment (covariance). Our approach is based on the efficient numerical integration of the Bayesian formula for optimal recursive filtering. The direct evaluation of the Jacobian matrix associated with the extended Kalman filter is avoided, which is similar to the one recently reported in [12]. Secondly, we use Gaussian sum filters for the development of nearly optimal filters. The Gaussian sum filter has been studied in $[1,20]$. However, we adapt our Gaussian filter for the update of Gaussian distributions. We also suggest some new update rules of weights for Gaussian sum filters. Through our experimental study we found that the filters developed in the paper perform better than or as good as the filter of Julier-Uhlmann [12]. They have a significant improvement over the extended Kalman filter with no additional cost.

An outline of the paper is as follows. In Section 2 we develop the Gaussian filter based on a single Gaussian distribution. In Section 3 we discuss the efficient numerical integration of the Gaussian filter based on quadrature rules, and introduce the Gauss-Hermite filter (GHF) and the central difference filter (CDF). In Section 4 we formulate the Gauss-Hermite filter and the central difference filter as the filter algorithms. In Section 5 we introduce the mixed Gaussian filter and the new update rules of weights. In Section 6 we discuss the relation of the Gaussian filter for the discrete time system to the continuous time optimal filter governed by the Zakai equation. In Section 7 we analyze the stability and performance bound of the Gaussian filters and the mixed Gaussian filters developed in the paper. In Section 8 we report our numerical findings and comparison studies. Also, we demonstrate a nearly optimal performance of the mixed Gaussian filter. Finally, we conclude our results in Section 9 .

\section{Gaussian Filters}

We discuss the nonlinear filtering problem for the discrete-time signal system for $R^{n}$-valued process $x(k)$ :

$$
x(k)=f(x(k-1))+w(k)
$$

and the observation process $y(k) \in R^{p}$ is given by

$$
y(k)=h(x(k))+v(k)
$$

where $w(k)$ and $v(k)$ are white noises with covariances $Q$ and $R$, respectively. We assume that the initial condition $x(0)$ and $w(k), v(k)$ are independent random variables. The optimal nonlinear filtering problem is to find the conditional expectation $E\left(x(k) \mid Y_{k}\right)$ of the process $x(k)$ given the observation data $Y_{k}=\{y(j), 1 \leq j \leq k\}$. The probability density function $p_{k \mid k}$ of the conditional expectation $E\left[x(k) \mid Y_{k}\right]$ is given by Bayes' formula

$$
p_{k \mid k-1}(x)=\int_{R^{n}} \frac{1}{\left((2 \pi)^{n} \operatorname{det} Q\right)^{1 / 2}} e^{-\frac{1}{2}(x-f(t))^{t} Q^{-1}(x-f(t))} p_{k-1 \mid k-1}(t) d t
$$


and

$$
p_{k \mid k}(x)=c e^{-\frac{1}{2}(y-h(x))^{t} R^{-1}(y-h(x))} p_{k \mid k-1}(x)
$$

where $p_{k \mid k-1}$ is the one-step prediction and is the probability density function of $x(k)$ conditioned on $Y_{k-1}$. That is, the recursive filter (2.3)-(2.4) consists of the prediction step (2.3) and the correction step (2.4). We consider a Gaussian approximation of this recursive formula and develop Gaussian filters. We assume that $p_{k-1 \mid k-1}$ is a single Gaussian distribution with mean $x_{k-1 \mid k-1}$ and covariance $P_{k-1 \mid k-1}$. Then we construct the Gaussian approximation of $p_{k \mid k}$ in the following two steps. First, we consider the predictor step. We approximate $p_{k \mid k-1}$ by the Gaussian distribution that has the same mean and covariance as $p_{k \mid k-1}$. By Fubini's theorem the mean and covariance of $p_{k \mid k-1}$ are given by

$$
\begin{aligned}
E_{k \mid k-1}[x] & =\int_{R^{n}} x p_{k \mid k-1}(x) d x=\int_{R^{n}}\left(\int_{R^{n}} \frac{1}{\left((2 \pi)^{n} \operatorname{det} Q\right)^{1 / 2}} e^{-\frac{1}{2}(x-f(t))^{t} Q^{-1}(x-f(t))} x d x\right) p_{k-1 \mid k-1}(t) d t \\
& =\int_{R^{n}} f(t) p_{k-1 \mid k-1}(t) d t
\end{aligned}
$$

and

$$
\begin{aligned}
& E_{k \mid k-1}\left[x x^{t}\right]=\int_{R^{n}} x x^{t} p_{k \mid k-1}(x) d x \\
& =\int_{R^{n}}\left(\int_{R^{n}} \frac{1}{\left((2 \pi)^{n} \operatorname{det} Q\right)^{1 / 2}} e^{-\frac{1}{2}(x-f(t))^{t} Q^{-1}(x-f(t))} x x^{t} d x\right) p_{k-1 \mid k-1}(t) d t \\
& =Q+\int_{R^{n}} f(t) f(t)^{t} p_{k-1 \mid k-1}(t) d t
\end{aligned}
$$

Remark 1.1: To derive the mean and covariance of $p_{k \mid k-1}$, we also can use (2.1) and the independence of $w(k)$ and $x(k-1)$. For example,

$$
E_{k \mid k-1}[x]=E\left[f(x(k-1))+w(k) \mid Y_{k-1}\right]=E\left[f(x(k-1)) \mid Y_{k-1}\right]
$$

which is precisely the same as the above, though differently expressed.

Thus, if $p_{k-1 \mid k-1}$ is a Gaussian with mean $x_{k-1 \mid k-1}$ and covariance $P_{k-1 \mid k-1}$, then the Gaussian approximation of $p_{k \mid k-1}$ has mean $x_{k \mid k-1}$ and covariance $P_{k \mid k-1}$ defined by

$$
x_{k \mid k-1}=\int_{R^{n}} f(t) \frac{1}{\left((2 \pi)^{n} \operatorname{det} P_{k-1 \mid k-1}\right)^{1 / 2}} e^{-\frac{1}{2}\left(t-x_{k-1 \mid k-1}\right)^{t} P_{k-1 \mid k-1}^{-1}\left(t-x_{k-1 \mid k-1}\right)} d t
$$

and

$$
\begin{aligned}
P_{k \mid k-1}= & Q+\int_{R^{n}}\left(f(t)-x_{k \mid k-1}\right)\left(f(t)-x_{k \mid k-1}\right)^{t} \frac{1}{\left((2 \pi)^{n} \operatorname{det} P_{k-1 \mid k-1}\right)^{1 / 2}} \\
& e^{-\frac{1}{2}\left(t-x_{k-1 \mid k-1}\right)^{t} P_{k-1 \mid k-1}^{-1}\left(t-x_{k-1 \mid k-1}\right)} d t .
\end{aligned}
$$

Next, we discuss the corrector step.

$$
p_{k \mid k}(x)=c e^{-\frac{1}{2}(y-h(x))^{t} R^{-1}(y-h(x))} p_{k \mid k-1}
$$


where $c$ is the normalization constant and we assume that $p_{k \mid k-1}$ is given by the Gaussian approximation defined by $(2.5)-(2.6)$. Define the innovation process

$$
I(k)=y(k)-E_{k \mid k-1}[y(k)]=y(k)-E_{k \mid k-1}[h(x(k)] .
$$

Here we again approximate for the conditional distribution of $(x(k), h(x(k))$ given the observations up to time $k-1$ by a Gaussian. That is, we approximate $E_{k \mid k-1}[h(x(k)]$ by its Gaussian approximation $z$. This means that the probability density function of $z$ is given by the Gaussian distribution with mean $\hat{z}$ and covariance $P_{z z}$ defined by

$$
\hat{z}=\int_{R^{n}} h(t) \frac{1}{\left((2 \pi)^{n} \operatorname{det} P_{k \mid k-1}\right)^{1 / 2}} e^{-\frac{1}{2}\left(t-x_{k \mid k-1}\right)^{t} P_{k \mid k-1}^{-1}\left(t-x_{k \mid k-1}\right)} d t
$$

and

$$
P_{z z}=\int_{R^{n}}(h(t)-\hat{z})(h(t)-\hat{z}) \frac{1}{\left((2 \pi)^{n} \operatorname{det} P_{k \mid k-1}\right)^{1 / 2}} e^{-\frac{1}{2}\left(t-x_{k \mid k-1}\right)^{t} P_{k \mid k-1}^{-1}\left(t-x_{k \mid k-1}\right)} d t .
$$

Finally, we construct the Gaussian approximation of $p_{k \mid k}$ with mean $x_{k \mid k}$ and covariance defined by

$$
x_{k \mid k}=x_{k \mid k-1}+L_{k}(y(k)-\hat{z})
$$

and

$$
P_{k \mid k}=P_{k \mid k-1}-L_{k} P_{x z}^{t}
$$

where the Kalman-filter gain $L_{k}$ is defined by

$$
L_{k}=P_{x z}\left(R+P_{z z}\right)^{-1}
$$

and the covariance $P_{x z}$ is defined by

$$
P_{x z}=\int_{R^{n}}\left(t-x_{k \mid k-1}\right)(h(t)-\hat{z})^{t} \frac{1}{\left((2 \pi)^{n} \operatorname{det} P_{k \mid k-1}\right)^{1 / 2}} e^{-\frac{1}{2}\left(t-x_{k \mid k-1}\right)^{t} P_{k \mid k-1}^{-1}\left(t-x_{k \mid k-1}\right)} d t .
$$

In order to implement the Gaussian filter we must develop the approximation methods to evaluate integrals (2.5)-(2.12). In Section 3 we discuss the approximation methods for the integration of the form

$$
\int_{R^{n}} F(t) \frac{1}{\left((2 \pi)^{n} \operatorname{det} \Sigma\right)^{1 / 2}} e^{-\frac{1}{2}(t-\bar{x})^{t} \Sigma^{-1}(t-\bar{x})} d t
$$

where $F(t)$ is a given function. Especially our discussions include the Gauss-Hermite quadrature rule and finite difference approximation. Note that a different approximation to this integration results in a variant of the Gaussian filter. For example, if we approximate the integration by linearizing $F$ at $\bar{x}$, then we obtain the extended Kalman filter. 
In order to improve the performance of Gaussian filter we also discuss the mixed Gaussian filter in Section 5. We approximate $p_{k-1 \mid k-1}$ by the linear combination of multiple Gaussian distributions, i.e.,

$$
p_{k-1 \mid k-1}(x)=\sum_{i=1}^{m} \alpha_{k-1}^{(i)} \frac{1}{\left((2 \pi)^{n} \operatorname{det} P_{k-1 \mid k-1}^{(i)}\right)^{1 / 2}} e^{-\frac{1}{2}\left(x-x_{k-1 \mid k-1}^{(i)}\right)^{t}\left(P_{k-1 \mid k-1}^{(i)}\right)^{-1}\left(x-x_{k-1 \mid k-1}^{(i)}\right)} .
$$

Then, each Gaussian distribution is updated separately by the Gaussian filter (2.5)-(2.12). In the corrector step we update the weights $\alpha_{k}^{(i)}$. For example, we can determine the weights $\alpha_{k}^{(i)}$ by the $L^{2}$-projection, i.e., $\alpha_{k}^{(i)}, 1 \leq i \leq m$, minimize

$$
\int_{R^{n}}\left|p_{k \mid k}(x)-\sum_{i=1}^{m} \alpha_{k}^{(i)} \frac{1}{\left((2 \pi)^{n} \operatorname{det} P_{k \mid k}^{(i)}\right)^{1 / 2}} e^{-\frac{1}{2}\left(x-x_{k \mid k}^{(i)}\right)^{t}\left(P_{k \mid k}^{(i)}\right)^{-1}\left(x-x_{k \mid k}^{(i)}\right)}\right|^{2} d x
$$

over $\left(R^{+}\right)^{m}$, where $p_{k \mid k}$ is defined as in the corrector step (2.4), assuming

$$
p_{k \mid k-1}(x)=\sum_{i=1}^{m} \alpha_{k-1}^{(i)} \frac{1}{\left((2 \pi)^{n} \operatorname{det} P_{k \mid k-1}^{(i)}\right)^{1 / 2}} e^{-\frac{1}{2}\left(x-x_{k \mid k-1}^{(i)}\right)^{t}\left(P_{k \mid k-1}^{(i)}\right)^{-1}\left(x-x_{k \mid k-1}^{(i)}\right)} .
$$

\section{Quadrature Rules}

In this section we discuss the approximation methods for the integral of the form

$$
I=\int_{R^{n}} F(t) \frac{1}{\left((2 \pi)^{n} \operatorname{det} \Sigma\right)^{1 / 2}} e^{-\frac{1}{2}(t-\bar{x})^{t} \Sigma^{-1}(t-\bar{x})} d t .
$$

If we assume $\Sigma=S^{t} S$ and change the coordinate of integration by $t=S^{t} s+\bar{x}$, then

$$
I=\int_{R^{n}} \tilde{F}(s) \frac{1}{(2 \pi)^{n / 2}} e^{-\frac{1}{2}|s|^{2}} d s
$$

with $\tilde{F}(s)=F\left(S^{t} s+\bar{x}\right)$. We apply the Gauss-Hermite quadrature rule. The Gauss-Hermite quadrature rule is given by

$$
\int_{-\infty}^{\infty} g(x) \frac{1}{(2 \pi)^{1 / 2}} e^{-x^{2}} d x=\sum_{i=1}^{m} w_{i} g\left(x_{i}\right)
$$

where the equality holds for all polynomials of degree up to $2 m-1$ and the quadrature points $x_{i}$ and the weights are determined (e.g., see [9]) as follows. Let $J$ be the symmetric tri-diagonal matrix with zero diagonals and $J_{i, i+1}=\sqrt{i / 2}, 1 \leq i \leq m-1$. Then $\left\{x_{i}\right\}$ are the eigenvalues of $J$ and $w_{i}$ equal to $\left|\left(v_{i}\right)_{1}\right|^{2}$ where $\left(v_{i}\right)_{1}$ is the first element of the $\mathrm{i}$-th normalized eigenvector of $J$. Thus, $I$ is approximated by

$$
I_{m}=\sum_{i_{1}=1}^{m} \ldots \sum_{i_{n}=1}^{m} \tilde{F}\left(q_{i_{1}}, q_{i_{2}}, \ldots, q_{i_{n}}\right) w_{i_{1}} w_{i_{2}} \ldots w_{i_{n}}
$$


where $q_{i}=\frac{x_{1}}{\sqrt{2}}, 1 \leq i \leq m$ and $I_{m}$ is exact for all polynomials of the form $s^{i_{1}} s^{i_{2}} \ldots s^{i_{n}}$ with $1 \leq i_{k} \leq 2 m-1$. In order to evaluate $I_{m}$ we need $m^{n}$-point function evaluations. For example $m=3$ we have

$$
q_{1}=-\sqrt{3}, q_{2}=0, q_{3}=\sqrt{3} \quad \text { and } \quad w_{1}=w_{3}=\frac{1}{6}, w_{2}=\frac{2}{3} .
$$

and $I_{3}$ requires 9 -point function evaluations for $n=2$.

In Julier-Uhlmann [12] the standard normal distribution is approximated by the discrete distribution as

$$
Z_{k}=\left\{\begin{array}{ll}
\sqrt{n+\kappa} e_{k}, & 1 \leq k \leq n \\
-Z_{k-n} & n+1 \leq k \leq 2 n \\
0 & k=2 n+1,
\end{array} \quad \text { and } \quad P\left(Z_{k}\right)= \begin{cases}\frac{2 \kappa}{2(n+\kappa)} & k=2 n+1 \\
\frac{1}{2(n+\kappa)} & 1 \leq k \leq 2 n\end{cases}\right.
$$

where $\kappa>0$ is a constant and $e_{k}$ is $k$-th unit vector in $R^{n}$. Here, this discrete distribution has the same first, second and higher odd moments as the standard normal distribution. Julier-Uhlmann developed a Gaussian filter based on the following quadrature rule for (3.1)

$$
I_{J U}=\sum_{k=1}^{2 n+1} \tilde{F}\left(Z_{k}\right) P\left(Z_{k}\right) .
$$

The JU-rule requires $(2 n+1)$-point function evaluation and is exact for all quadratic polynomials. If we set $\kappa=2$ and $n=1$, then the JU-rule coincides with the Gauss-Hermite rule $I_{3}$.

Finally we consider the polynomial interpolation methods. We approximate $\tilde{F}(t)$ by the quadratic function $P_{2}$ that satisfies

$$
\tilde{F}\left(s_{i}\right)=P_{2}\left(s_{i}\right), \quad 1 \leq i \leq \frac{(n+1)(n+2)}{2}
$$

at the points $\left\{s_{i}\right\}$ in $R^{n}$ consisting of

$$
0, h e_{i}, \quad-h e_{i}, 1 \leq i \leq n \text { and } h e_{i}+h e_{j}, 1 \leq i<j \leq n,
$$

where $h>0$ is the stepsize. That is, $P_{2}$ is given by

$$
P_{2}(s)=\tilde{F}(0)+\sum_{i=1}^{n} a_{i} s_{i}+\frac{1}{2} s^{t} H s
$$

where $s_{i}$ is the $i$-th coordinate of a point $s \in R^{n}, a_{i}$ and the symmetric matrix $H=\left(H_{i, j}\right)_{n \times n}$ 
are defined by

$$
\begin{aligned}
& a_{i}=\frac{\tilde{F}\left(h e_{i}\right)-\tilde{F}\left(-h e_{i}\right)}{2 h}, 1 \leq i \leq n \\
& H_{i, i}=\frac{\tilde{F}\left(h e_{i}\right)-2 \tilde{F}(0)+\tilde{F}\left(-h e_{i}\right)}{h^{2}}, \quad 1 \leq i \leq n \\
& H_{i, j}=\frac{\tilde{F}\left(h e_{i}+h e_{j}\right)-\tilde{F}\left(-h e_{i}\right)-\tilde{F}\left(-h e_{j}\right)+\tilde{F}(0)}{h^{2}}, 1 \leq i<j \leq n .
\end{aligned}
$$

Thus, we approximate (3.1) by

$$
I_{q}=\int_{R^{n}} P_{2}(s) \frac{1}{(2 \pi)^{n / 2}} e^{-\frac{1}{2}|s|^{2}} d s=\tilde{F}(0)+\sum_{i=1}^{n} \frac{1}{2} H_{i, i}
$$

and the integral

$$
J=\int_{R^{n}} \tilde{F}_{1}(s) \tilde{F}_{2}(s) \frac{1}{(2 \pi)^{n / 2}} e^{-\frac{1}{2}|s|^{2}} d s
$$

by

$$
\begin{aligned}
J_{q}= & \int_{R^{n}} P_{2}^{(1)}(s) P_{2}^{(2)}(s) \frac{1}{(2 \pi)^{n / 2}} e^{-\frac{1}{2}|s|^{2}} d s \\
& =I_{q}^{(1)} I_{q}^{(2)}+\sum_{i=1}^{n}\left(a_{i}^{(1)} a_{i}^{(2)}+\frac{1}{2} H_{i, i}^{(1)} H_{i, i}^{(2)}\right)+\sum_{i \neq j} H_{i, j}^{(1)} H_{i, j}^{(2)}
\end{aligned}
$$

where $P_{2}^{(i)}$ is the quadratic approximation of $\tilde{F}_{i}$ for $i=1,2$.

Next, we consider the approximation $P_{1}$ of $\tilde{F}$, defined by

$$
P_{1}(s)=\tilde{F}(0)+\sum_{i=1}^{n} \frac{\tilde{F}\left(h e_{i}\right)-\tilde{F}\left(-h e_{i}\right)}{2 h} s_{i}+\sum_{i=1}^{n} \frac{1}{2} H_{i, i} s_{i}^{2}
$$

which only bases on the values $\tilde{F}\left( \pm h e_{i}\right)=P_{1}\left( \pm h e_{i}\right), 1 \leq i \leq n$ and $\tilde{F}(0)=P_{1}(0)$, and uses the diagonal second order correction $\sum_{i=1}^{n} \frac{1}{2} H_{i i} s_{i}^{2}$ of the central difference approximation of $\tilde{F}$ in $(3.5)$. Then the integrals $I$ and $J$ are approximated by

$$
I_{c}=\int_{R^{n}} P_{1}(s) \frac{1}{(2 \pi)^{n / 2}} e^{-\frac{1}{2}|s|^{2}} d s=\tilde{F}(0)+\sum_{i=1}^{n} \frac{1}{2} H_{i, i}
$$

and

$$
\begin{aligned}
J_{c}= & \int_{R^{n}} P_{1}^{(1)}(s) P_{1}^{(2)}(s) \frac{1}{(2 \pi)^{n / 2}} e^{-\frac{1}{2}|s|^{2}} d s \\
& =\sum_{i=1}^{n} \frac{\tilde{F}_{1}\left(h e_{i}\right)-\tilde{F}_{1}\left(-h e_{i}\right)}{2 h} \frac{\tilde{F}_{2}\left(h e_{i}\right)-\tilde{F}_{2}\left(-h e_{i}\right)}{2 h}+\tilde{F}_{1}(0) \tilde{F}_{2}(0)+\sum_{i=1}^{n} \frac{1}{2} H_{i, i}^{(1)} H_{i, i}^{(2)} .
\end{aligned}
$$

If we remove the second order correction term in $(3.9)-(3.10)$, then this coincides with the extended Kalman filter with the central difference approximation of the Jacobian of $\tilde{F}$. 


\section{Filter Algorithms}

In this section we describe the filter algorithms that are based on the Gaussian filter and quadrature methods described in Section 3. We first consider the Gauss-Hermite filter based on the Gaussian-Hermite quadrature rule. Let $\left(q_{i}, w_{i}\right)$ be the quadrature rule for

$$
\int_{R^{n}} F(x) \frac{1}{(2 \pi)^{n / 2}} e^{-|x|^{2}} d x \sim \sum_{i=1}^{N} w_{i} F\left(q_{i}\right) .
$$

Let $\hat{x}_{0}$ and $P_{0}$ be the starting values for the mean and covariance of the random variable $x(0)$ and set $P_{0 \mid 0}=P_{0}$ and $x_{0 \mid 0}=\hat{x}_{0}$. In Gaussian-Hermite filter we apply the following predictor and corrector steps recursively.

\section{Gauss-Hermite Filter}

Predictor Step Compute the factorization $P_{k-1 \mid k-1}=S^{t} S$ and set $x_{i}=S^{t} q_{i}+x_{k-1 \mid k-1}$. Update $p_{k \mid k-1}=N\left(x_{k \mid k-1}, P_{k \mid k-1}\right)$ by

$$
\begin{aligned}
& x_{k \mid k-1}=\sum_{i=1}^{N} f\left(x_{i}\right) w_{i} \\
& P_{k \mid k-1}=Q+\sum_{i=1}^{N}\left(f\left(x_{i}\right)-x_{k \mid k-1}\right)\left(f\left(x_{i}\right)-x_{k \mid k-1}\right)^{t} w_{i} .
\end{aligned}
$$

Corrector Step Compute the factorization $P_{k \mid k-1}=T^{t} T$ and set $x_{i}=T^{t} q_{i}+x_{k \mid k-1}$. Update $p_{k \mid k}=N\left(x_{k \mid k}, P_{k \mid k}\right)$ by

$$
\begin{aligned}
& x_{k \mid k}=x_{k \mid k-1}+L_{k}\left(y(k)-z_{k}\right) \\
& P_{k \mid k}=P_{k \mid k-1}-L_{k} P_{x z}^{t}
\end{aligned}
$$

where

$$
\begin{aligned}
& z_{k}=\sum_{i=1}^{N} h\left(x_{i}\right) w_{i} \\
& P_{x z}=\sum_{i=1}^{N}\left(x_{i}-x_{k \mid k-1}\right)\left(h\left(x_{i}\right)-z_{k}\right)^{t} w_{i} . \\
& P_{z z}=\sum_{i=1}^{N}\left(h\left(x_{i}\right)-z_{k}\right)\left(h\left(x_{i}\right)-z_{k}\right)^{t} w_{i} . \\
& L_{k}=P_{x z}\left(R+P_{z z}\right)^{-1} .
\end{aligned}
$$

We use the Choresky decomposition for finding $S$ and $T[6]$ in our calculations. One of the advantages of the quadrature based filter is that we do not require to have the derivative of $f$ and $h$.

Secondly, we present the filter algorithm based on the central difference approximation with second order diagonal correction. We summarize the filter algorithm of the central difference approximation with second order diagonal correction as follows.

\section{Central Difference Filter}


Predictor Step Compute the factorization $P_{k-1 \mid k-1}=S^{t} S$ and set $\tilde{f}(s)=f\left(S^{t} s+x_{k-1 \mid k-1}\right)$ approximated by $\tilde{f}(0)+\sum_{i=1}^{n} a_{i} s_{i}+\frac{1}{2} s^{t} H s$. Update $p_{k \mid k-1}=N\left(x_{k \mid k-1}, P_{k \mid k-1}\right)$ by

$$
\begin{aligned}
& x_{k \mid k-1}=\tilde{f}(0)+\sum_{i=1}^{n} \frac{1}{2} H_{i, i} \\
& P_{k \mid k-1}=Q+\sum_{i=1}^{n} a_{i} a_{i}^{t}+\sum_{i=1}^{n} \frac{1}{2} H_{i, i} H_{i, i}^{t}
\end{aligned}
$$

where $H_{i, i}$ are the central difference approximations of $\frac{\partial^{2} \tilde{f}(s)}{\partial s_{i}^{2}}$.

Corrector Step Compute the factorization $P_{k \mid k-1}=T^{t} T$ and set $\tilde{h}(s)=h\left(T^{t} s+x_{k \mid k-1}\right)$ approximated by $\tilde{h}(0)+\sum_{i=1}^{n} b_{i} s_{i}+\frac{1}{2} s^{t} G s$. Update $p_{k \mid k}=N\left(x_{k \mid k}, P_{k \mid k}\right)$ by

$$
\begin{aligned}
& x_{k \mid k}=x_{k \mid k-1}+L_{k}\left(y(k)-z_{k}\right) \\
& P_{k \mid k}=P_{k \mid k-1}-L_{k} P_{x z}^{t}
\end{aligned}
$$

where

$$
\begin{aligned}
& z_{k}=\tilde{h}(0)+\sum_{i=1}^{n} \frac{1}{2} G_{i, i} \\
& P_{x z}=T^{t}\left(b_{1}, \cdots, b_{n}\right)^{t} . \\
& P_{z z}=\sum_{i=1}^{n} b_{i} b_{i}^{t}+\sum_{i=1}^{n} \frac{1}{2} G_{i, i} G_{i, i}^{t} . \\
& L_{k}=P_{x z}\left(R+P_{z z}\right)^{-1} .
\end{aligned}
$$

where $G_{i, i}$ are the central difference approximations of $\frac{\partial^{2} \tilde{h}(s)}{\partial s_{i}^{2}}$.

In the algorithm we avoid to calculate the derivatives of $f$ and $h$. Instead, we use the central difference.

The followings are the operation counts in terms of function evaluations.

If we use the quadrature rule based on the $q$-point Gauss-Hermite rule then the algorithm requires $(n+p) q^{n}$ function evaluations.

If we use the Julier-Uhlmann discrete Gaussian rule then the algorithm requires $(n+p)(2 n+1)$ function evaluations.

If we use the central difference algorithm, the $(n+p)(2 n+1)$ function evaluation are required.

As we will see, our numerical testings indicate that the algorithm based on the GaussHermite rule performs better than the others. If we use the 3 -point rule, then we require $729(n+p)$ function evaluations for the case $n=6$, which can be done in real-time. 


\section{$5 \quad$ Mixed Gaussian Filter}

In this section we discuss the mixed Gaussian filter. We approximate the conditional probability density $p_{k-1 \mid k-1}$ by the linear combination of multiple Gaussian distributions, i.e.,

$$
p_{k-1 \mid k-1}(x)=\sum_{i=1}^{m} \alpha_{k-1}^{(i)} \frac{1}{\left((2 \pi)^{n} \operatorname{det} P_{k-1 \mid k-1}^{(i)}\right)^{1 / 2}} e^{-\frac{1}{2}\left(x-x_{k-1 \mid k-1}^{(i)}\right)^{t}\left(P_{k-1 \mid k-1}^{(i)}\right)^{-1}\left(x-x_{k-1 \mid k-1}^{(i)}\right)} .
$$

Here we apply the Gaussian filter (2.5)-(2.12) to each Gaussian distribution $N\left(x_{k-1 \mid k-1}^{(i)}, P_{k-1 \mid k-1}^{(i)}\right)$ and obtain the update $N\left(x_{k \mid k}^{(i)}, P_{k \mid k}^{(i)}\right)$. Each update is independent from the others and can be performed in a parallel manner.

Next, we update the weights $\alpha_{k}^{(i)}$ for the new update $p_{k \mid k}(x)$ at the end of corrector step. We discuss here three update formulae in what follows.

First, by equating the first moment of each Gaussian distribution we obtain

$$
\begin{aligned}
& \alpha_{k}^{(i)} \int_{R^{n}} \frac{1}{\left((2 \pi)^{n} \operatorname{det} P_{k \mid k}^{(i)}\right)^{1 / 2}} e^{-\frac{1}{2}\left(x-x_{k \mid k}^{(i)}\right)^{t}\left(P_{k \mid k}^{(i)}\right)^{-1}\left(x-x_{k \mid k}^{(i)}\right)} d x \\
& \quad=\alpha_{k-1}^{(i)} \int_{R^{n}} \frac{1}{(2 \pi)^{n}\left(\operatorname{det} R \operatorname{det} P_{k \mid k-1}^{(i)}\right)^{1 / 2}} e^{\left.-\frac{1}{2}(y-h(x))^{t} R^{-1}(y-h(x))+\left(x-x_{k \mid k-1}^{(i)}\right)^{t}\left(P_{k \mid k-1}^{(i)}\right)^{-1}\left(x-x_{k \mid k-1}^{(i)}\right)\right)} d x .
\end{aligned}
$$

Here we approximate the right hand side by the Gaussian distribution as in (2.5)-(2.6) and obtain

$$
\alpha_{k}^{(i)}=\alpha_{k-1}^{(i)} \frac{1}{\left((2 \pi)^{n} \operatorname{det}\left(R+P_{z z}\right)\right)^{1 / 2}} e^{-\frac{1}{2}(y-\hat{z})^{t}\left(R+P_{z z}\right)^{-1}(y-\hat{z})}
$$

where $\hat{z}, P_{z z}$ are defined by (2.7)-(2.8), which is the update formula discussed in [1].

Next, we apply the collocation condition at $x_{k \mid k}^{(i)}$ :

$$
\begin{aligned}
& \alpha_{k}^{(i)} \frac{1}{\left((2 \pi)^{n} \operatorname{det} P_{k \mid k}^{(i)}\right)^{1 / 2}} \\
& \quad=\alpha_{k-1}^{(i)} \frac{1}{\left((2 \pi)^{n} \operatorname{det} P_{k \mid k-1}^{(i)}\right)^{1 / 2}} e^{-\frac{1}{2}\left(\left(y-h\left(x_{k \mid k}^{(i)}\right)\right)^{t} R^{-1}\left(y-h\left(x_{k \mid k}^{(i)}\right)\right)+\left(x_{k \mid k}^{(i)}-x_{k \mid k-1}^{(i)}\right)^{t}\left(P_{k \mid k-1}^{(i)}\right)^{-1}\left(x_{k \mid k}^{(i)}-x_{k \mid k-1}^{(i)}\right)\right)}
\end{aligned}
$$

to obtain the update

$$
\alpha_{k}^{(i)}=\alpha_{k-1}^{(i)} \frac{\left(\operatorname{det} P_{k \mid k}^{(i)}\right)^{1 / 2}}{\left(\operatorname{det} P_{k \mid k-1}^{(i)}\right)^{1 / 2}} e^{-\frac{1}{2}\left(\left(y-h\left(x_{k \mid k}^{(i)}\right)\right)^{t} R^{-1}\left(y-h\left(x_{k \mid k}^{(i)}\right)\right)+\left(x_{k \mid k}^{(i)}-x_{k \mid k-1}^{(i)}\right)^{t}\left(P_{k \mid k-1}^{(i)}\right)^{-1}\left(x_{k \mid k}^{(i)}-x_{k \mid k-1}^{(i)}\right)\right)} .
$$

Finally we discuss the simultaneous update of the weights. We determine the weights $\alpha_{k}^{(i)}$ by the $L^{2}$-projection, i.e., $\alpha_{k}^{(i)}, 1 \leq i \leq m$ minimize

$$
\int_{R^{n}}\left|p_{k \mid k}(x)-\sum_{i=1}^{m} \alpha_{k}^{(i)} \frac{1}{\left((2 \pi)^{n} \operatorname{det} P_{k \mid k}^{(i)}\right)^{1 / 2}} e^{-\frac{1}{2}\left(x-x_{k \mid k}^{(i)}\right)^{t}\left(P_{k \mid k}^{(i)}\right)^{-1}\left(x-x_{k \mid k}^{(i)}\right)}\right|^{2} d x
$$


over $\left(R^{+}\right)^{m}$, where $p_{k \mid k}$ is defined as in the corrector step (2.4) with

$$
p_{k \mid k-1}(x)=\sum_{i=1}^{m} \alpha_{k-1}^{(i)} \frac{1}{\left((2 \pi)^{n} \operatorname{det} P_{k \mid k-1}^{(i)}\right)^{1 / 2}} e^{-\frac{1}{2}\left(x-x_{k \mid k-1}^{(i)}\right)^{t}\left(P_{k \mid k-1}^{(i)}\right)^{-1}\left(x-x_{k \mid k-1}^{(i)}\right)} .
$$

In order to perform the minimization (5.4) we need to evaluate the integral of the form

$$
\int_{R^{n}} e^{-\frac{1}{2}(y-h(x))^{t} R^{-1}(y-h(x))} \frac{1}{\left((2 \pi)^{n} \operatorname{det} \Sigma\right)^{1 / 2}} e^{-\frac{1}{2}(x-\bar{x})^{t} \Sigma^{-1}(x-\bar{x})} d x .
$$

and it is relatively expensive. Hence we propose the minimization of the sum of collocation distances:

$$
\sum_{i=1}^{m}\left|p_{k \mid k}\left(x_{k \mid k}^{(i)}\right)-\sum_{j=1}^{m} \alpha_{k}^{(j)} \frac{1}{\left((2 \pi)^{n} \operatorname{det} P_{k \mid k}^{(j)}\right)^{1 / 2}} e^{-\frac{1}{2}\left(x_{k \mid k}^{(i)}-x_{k \mid k}^{(j)}\right)^{t}\left(P_{k \mid k}^{(j)}\right)^{-1}\left(x_{k \mid k}^{(i)}-x_{k \mid k}^{(j)}\right)}\right|^{2} .
$$

over $\alpha \in R^{m}$ satisfying $\alpha \geq \alpha_{0}>0$. A positive constant $\alpha_{0}$ is chosen so that the likelihood of each Gaussian distribution is nonzero (e.g., $\alpha_{0}=0.001(1, \ldots, 1)^{t} \in R^{m}$ ). Problem (5.5) is formulated as the quadratic programming

$$
\min \frac{1}{2} \alpha^{t} A^{t} A \alpha-A^{t} b+\frac{\delta}{2}|\alpha|^{2} \quad \text { subject to } \alpha \geq \alpha_{0},
$$

where $\delta>0$ is chosen so that the singularity of the matrix $A^{t} A$ is avoided and the matrices $(A, b)$ are defined by

$$
\begin{aligned}
& A_{i, j}=\frac{1}{\left((2 \pi)^{n} \operatorname{det} P_{k \mid k}^{(i)}\right)^{1 / 2}} e^{-\frac{1}{2}\left(x_{k \mid k}^{(i)}-x_{k \mid k}^{(j)}\right)^{t}\left(P_{k \mid k}^{(i)}\right)^{-1}\left(x_{k \mid k}^{(i)}-x_{k \mid k}^{(j)}\right)} \\
& b_{i}=\sum_{j=1}^{m} \frac{1}{(2 \pi)^{n}\left(\operatorname{det} R \operatorname{det} P_{k \mid k-1}^{(j)}\right)^{1 / 2}} e^{-\frac{1}{2}\left(\left(y-h\left(x_{k \mid k}^{(i)}\right)\right)^{t} R^{-1}\left(y-h\left(x_{k \mid k}^{(i)}\right)\right)+\left(x_{k \mid k}^{(i)}-x_{k \mid k-1}^{(i)}\right)^{t}\left(P_{k \mid k-1}^{(j)}\right)^{-1}\left(x_{k \mid k}^{(i)}-x_{k \mid k-1}^{(j)}\right)\right)} .
\end{aligned}
$$

Thus, we solve (5.6) to obtain the weights $\alpha_{k}^{(j)}$ at each corrector step by using the existing numerical optimization method (e.g., see [6]).

The theoretical foundation of the Gaussian sum approximation as above is that any probability density function can be approximated as closely as desired by a Gaussian sum. More precisionly, we state the following error estimate, the proof of which is found in [17].

Theorem 5.1: Let $\mathcal{M}$ be a non-negative integer and $\mathcal{N}=2 \mathcal{M}+2$. For any $\epsilon>0$ there must exist $D>0$ and a mask

$$
\left\{\alpha_{j}\left|j=\left(j_{1}, \ldots, j_{n}\right)^{t} \in Z^{n},\right| j \mid=\sum_{s=1}^{n} j_{s} \leq \mathcal{M}\right\}
$$

such that for any density function $p(x) \in C^{\mathcal{N}}\left(R^{n}\right) \cap W_{\infty}^{\mathcal{N}}\left(R^{n}\right)$ and all $h>0$ the estimate

$$
\left\|p(x)-p_{h}(x)\right\| \leq c_{\mathcal{N}} h^{\mathcal{N}}|p|_{W_{\infty}^{\mathcal{N}}\left(R^{n}\right)}+\epsilon\|p\|_{W_{\infty}^{\mathcal{N}-1}\left(R^{n}\right)}
$$


holds, where $p_{h}(x)$ is a linear combination of Gaussian distributions given by

$$
p_{h}(x)=\frac{1}{\sqrt{(2 \pi)^{n} D h^{2}}} \sum_{k \in Z^{n}} p_{k} e^{-\frac{|x-h k|^{2}}{2 D h^{2}}}
$$

with

$$
p_{k}=\sum_{|j| \leq \mathcal{M}} \hat{\alpha}_{j} p(h(k-j)), \quad \hat{\alpha}_{j}=h D^{-\frac{n-1}{2}} \alpha_{j}
$$

and $c_{\mathcal{N}}$ is a constant independent of $p(x)$. Here $|\cdot|_{W_{\infty}^{\mathcal{N}}\left(R^{n}\right)}$ and $\|\cdot\|_{W_{\infty}^{\mathcal{N}-1}\left(R^{n}\right)}$ are defined by

$$
|p|_{W_{\infty}^{\mathcal{N}}\left(R^{n}\right)}=\sum_{|j|=\mathcal{N}}\left\|\partial^{j} p\right\|_{C\left(R^{n}\right)}
$$

and

$$
\|p\|_{W_{\infty}^{\mathcal{N}-1}\left(R^{n}\right)}=\sum_{|j|=0}^{\mathcal{N}-1} \frac{\left|\partial^{j} p\right|}{j !}
$$

respectively.

Roughly speaking, the estimate (5.7) shows that any probability density function $p(x) \in$ $C^{\mathcal{N}}\left(R^{n}\right) \cap W_{\infty}^{\mathcal{N}}\left(R^{n}\right)$ can be approximated by a sum of Gaussian distributions each of whose components is given by

$$
\frac{1}{\sqrt{(2 \pi)^{n} D h^{2}}} e^{-\frac{|x-h k|^{2}}{2 D h^{2}}}
$$

with order $O\left(h^{\mathcal{N}}\right)$ for $h \rightarrow 0$.

\section{Relation to Continuous-Time Filter}

In this section let us discuss the nonlinear filtering problem for the continuous-time signal process $x(t)$ in $R^{n}$ generated by

$$
d x(t)=f(x(t)) d t+Q^{1 / 2} d B(t) .
$$

where $B(t)$ is the standard Brownian motion, i.e., $x(t)$ is the diffusion process $[3,11,19]$. So, equation (6.1) holds in the sense of Ito and $x(t)$ satisfies

$$
x(t)=x(s)+\int_{s}^{t} f(x(\tau)) d \tau+Q^{1 / 2}(B(t)-B(s))
$$

for all $0 \leq s \leq t$.

Now we consider the continuous observation process $z(t) \in R^{p}$

$$
z(t)=\int_{0}^{t} h(x(s)) d s+R^{1 / 2} V(t)
$$


where $V(t)$ is the standard Brownian process that is independent of $B(t)$ and $x(0)$.

Or, we consider the discrete observation process $y(k) \in R^{p}$

$$
y(k)=h(x(k \Delta t))+v(k) .
$$

where $\Delta t>0$ is the stepsize and $v(k)$ is white noise with covariance $R$. We assume that the initial condition $x(0)$ and $B(t), v(k)$ are independent.

Then the conditional probability density $p(t)=p(t, x) \in L^{2}\left(R^{n}\right)$ satisfies the Zakai equation

$$
d p(t)=A p(t) d t+R^{-1} h p d z(t)
$$

where $A$ is the Fokker-Planck operator [3, 19].

As shown in $[8,10,15]$, the discrete-time filter (2.3)-(2.4) applied to the time-discretized signal system of $(6.1)-(6.2)$ :

$$
\begin{aligned}
& x(k)=F(x(k-1))+Q^{1 / 2}(B(k \Delta t)-B((k-1) \Delta t)) \\
& y(k)=H(x(k))+R^{1 / 2}(V(k \Delta t)-V((k-1) \Delta t))
\end{aligned}
$$

provides an approximation method for the continuous-time optimal filter to (6.1)-(6.2). Note that $F(s)$ denotes the finite difference approximation

$$
x \simeq F(s)=s+\Delta t f(s)
$$

for the ordinary differential equation:

$$
\frac{d x}{d t}=f(x), \quad x(0)=s, \quad s \in R^{n} .
$$

Next, we apply the discrete-time filter in the following manner. We discuss the continuoustime process (6.1) with the discrete-time observation (6.3). We approximate the process $x(t)$ by the discrete-time process $x_{k}^{j}$ by

$$
x_{k}^{j}=\Phi\left(\delta, x_{k}^{j-1}\right)+Q^{1 / 2} w_{k}^{j}, \quad 1 \leq j \leq M
$$

with

$$
w_{k}^{j}=B(k \Delta t+j \delta)-B(k \Delta t+(j-1) \delta) .
$$

Here the interval $[k \Delta t,(k+1) \Delta t]$ is subdivided into the $M$ subintervals

$$
[k \Delta t+(j-1) \delta, k \Delta t+j \delta]
$$

where $1 \leq j \leq M$ and $\delta=\frac{\Delta t}{M}$.

So, $x_{k}^{j}$ denotes the approximation of the process $x(t)$ at $t=k \Delta t+j \delta$ and $\Phi\left(\delta, x_{k}^{j-1}\right)$ denotes the discretization of the ordinary differential equation

$$
\frac{d x}{d t}=f(x(t)) \text {. }
$$


We employ a family of Runge-Kutta methods in our calculation, including the Euler approximation:

$$
\Phi\left(\delta, x_{k}^{j-1}\right)=x_{k}^{j-1}+\delta f\left(x_{k}^{j-1}\right)
$$

The predictor step (2.5)-(2.6) is applied successively $M$ times to obtain $\left(x_{k \mid k-1}, P_{k \mid k-1}\right)$, i.e.,

$$
x_{k \mid k-1}^{j}=\int_{R^{n}} f(t) \frac{1}{\left((2 \pi)^{n} \operatorname{det} P_{k \mid k-1}^{j-1}\right)^{1 / 2}} e^{-\frac{1}{2}\left(t-x_{k \mid k-1}^{j-1}\right)^{t}\left(P_{k \mid k-1}^{j-1}\right)^{-1}\left(t-x_{k \mid k-1}^{j-1}\right)} d t
$$

and

$$
\begin{aligned}
P_{k \mid k-1}^{j}= & +\int_{R^{n}}\left(f(t)-x_{k \mid k-1}^{j}\right)\left(f(t)-x_{k \mid k-1}^{j}\right)^{t} \frac{1}{\left((2 \pi)^{n} \operatorname{det} P_{k \mid k-1}^{j-1}\right)^{1 / 2}} \\
& \times e^{-\frac{1}{2}\left(t-x_{k \mid k-1}^{j-1}\right)^{t}\left(P_{k \mid k-1}^{j-1}\right)^{-1}\left(t-x_{k \mid k-1}^{j-1}\right)} d t
\end{aligned}
$$

with

$$
x_{k \mid k-1}^{0}=x_{k-1 \mid k-1}, \quad P_{k \mid k-1}^{0}=P_{k-1 \mid k-1}
$$

for $1 \leq j \leq M$. Hence, the mean and covariance of $p_{k \mid k-1}$ are given by

$$
x_{k \mid k-1}=x_{k \mid k-1}^{M}, \quad P_{k \mid k-1}=P_{k \mid k-1}^{M} .
$$

Then the corrector step (2.7)-(2.12) is applied as it is to obtain $\left(x_{k \mid k}, P_{k \mid k}\right)$.

\section{Stability Analysis of Filter Algorithms}

In this section we analyze the stability and performance bound of the Gaussian filters (2.5)(2.12) for the signal process (6.5) with $F(s)=s+\Delta t f(s)$, i.e., a discrete approximation of the continuous signal system $(6.1)$ can be given by

$$
x(k)=x(k-1)+\Delta t f(x(k-1))+\sqrt{\Delta t} w(k)
$$

where $w(k)$ is the white noise with covariance $Q$.

We assume that

$$
\left|f\left(x_{1}\right)-f\left(x_{2}\right), x_{1}-x_{2}\right| \leq \omega\left|x_{1}-x_{2}\right|^{2}, \quad \text { for } \quad x_{1}, x_{2} \in R^{n} .
$$

Then we have the following theorem.

Theorem 7.1: The solution $P_{k \mid k}$ to $(2.5)-(2.12)$ has the estimate

$$
\operatorname{tr} P_{k \mid k} \leq(1+\omega \Delta t)^{2 k}\left(\operatorname{tr} P_{0 \mid 0}+\frac{\Delta t}{(1+\omega \Delta t)^{2}-1} \operatorname{tr} Q\right), \quad k \geq 0
$$


Proof: From (2.5)-(2.6) the Gaussian approximation of $p_{k \mid k-1}$ has the mean $x_{k \mid k-1}$ and covariance $P_{k \mid k-1}$ defined by

$$
x_{k \mid k-1}=\int_{R^{n}} F(s) p_{k-1 \mid k-1} d s
$$

and

$$
P_{k \mid k-1}=\Delta t Q+\int_{R^{n}}\left(F(s)-x_{k \mid k-1}\right)\left(F(s)-x_{k \mid k-1}\right)^{t} p_{k-1 \mid k-1}(s) d s .
$$

Note that

$$
\begin{aligned}
F(t) & -x_{k \mid k-1}=\int_{R^{n}}(F(t)-F(s)) p_{k-1 \mid k-1}(s) d s \\
& =\int_{R^{n}}(t-s+\Delta t(f(t)-f(s))) p_{k-1 \mid k-1}(s) d s
\end{aligned}
$$

Thus by the Cauchy and Schwarz inequality we have

$$
\begin{aligned}
& \operatorname{tr}\left[\left(F(t)-x_{k \mid k-1}\right)\left(F(t)-x_{k \mid k-1}\right)^{t}\right]= \\
& \quad \leq \int_{R^{n}}|t-s+\Delta t(f(t)-f(s))|^{2} p_{k-1 \mid k-1}(s) d s .
\end{aligned}
$$

Hence it follows from (7.3) that

$$
\begin{aligned}
& \operatorname{tr} P_{k \mid k-1}=\Delta t(\operatorname{tr} Q)+\operatorname{tr}\left[\int_{R^{n}} \int_{R^{n}}|t-s+\Delta t(f(t)-f(s))|^{2} p_{k-1 \mid k-1}(s) p_{k-1 \mid k-1}(t) d s d t\right] \\
& \leq \Delta t(\operatorname{tr} Q)+\operatorname{tr} P_{k-1 \mid k-1}+2 \Delta t \omega \operatorname{tr} P_{k-1 \mid k-1}+\Delta t^{2} \omega^{2} \operatorname{tr} P_{k-1 \mid k-1}
\end{aligned}
$$

by assumption (7.1). That is,

$$
\operatorname{tr} P_{k \mid k-1} \leq\left(1+2 \Delta t \omega+\Delta t^{2} \omega^{2}\right) \operatorname{tr} P_{k-1 \mid k-1}+\Delta t(\operatorname{tr} Q)
$$

Note that for any $k$ the covariance $P_{k \mid k}$ is nonegative and satisfies the following property

$$
P_{k \mid k} \leq P_{k \mid k-1} .
$$

Thus by (7.5) we obtain the estimate (7.2).

Remark 7.1: Using the similar arguments that lead to (7.2), we can prove that the same result holds for the approximation method discussed in Sections 3-4. That is, the estimate (7.2) is still valid for the Gaussian-Hermite filter, provided that $\sum_{i=1}^{N} w_{i}=1, w_{i} \geq 0$.

Next, we discuss the stability of the mixed Gaussian filter. The mixed Gaussian filter assumes the form

$$
p_{k \mid k}(x)=\sum_{j=1}^{m} \alpha_{k}^{(j)} N\left(x_{k \mid k}^{(j)}, P_{k \mid k}^{(j)}\right)(x)
$$


where the $j$-th Gaussian distribution $N\left(x_{k \mid k}^{(j)}, P_{k \mid k}^{(j)}\right)(x)$ is computed by the Gaussian filters described in Sections 3-4 in a parallel manner. The weights $\alpha_{k}^{(j)}, 1 \leq j \leq m$ are determined at the end of the corrector step based on the likelihood of each Gaussian distribution. Under the assumption (7.1) each Gaussian filter in the Gaussian sum (7.6) is stable in the sense of (7.2).

Next we discuss the performance bound. In general, let the operators $\mathcal{P}_{k \mid k-1}(\cdot)$ and $\Psi_{k \mid k-1}(\cdot)$ denote the one-step update of conditional probability density functions that are based on the exact filter (2.3)-(2.4) and the mixed Gaussian filter discussed in Section 5, respectively. We estimate the difference between the exact filter and the mixed Gaussian filter:

$$
\mathcal{E}_{n}=\prod_{k=1}^{n} \mathcal{P}_{k \mid k-1}\left(p_{0}(x)\right)-\Pi_{k=1}^{n} \Psi_{k \mid k-1}\left(\sum_{j=1}^{m} \alpha_{j} p_{0 \mid 0}^{(j)}(x)\right)
$$

where $p_{0}(x)$ is the probability density function of random variable $x(0)$ and

$$
p_{0 \mid 0}=\sum_{j=1}^{m} \alpha_{j} p_{0 \mid 0}^{(j)}
$$

is the mixed Gaussian approximation of $p_{0}(x)$. Let $|f|=\int_{R^{n}}|f| d x$. Then we have the following result.

Theorem 7.2: Assume that for any probability density function $p(x)$ and $q(x)$

$$
\left|\Psi_{n \mid n-1}(p)-\Psi_{n \mid n-1}(q)\right| \leq \gamma|p-q|
$$

Then the error estimate

$$
\left|\mathcal{E}_{n}\right| \leq \gamma^{n}\left|\mathcal{E}_{0}\right|+\sum_{j=1}^{n} \gamma^{n-j} \varepsilon_{j-1}
$$

holds, where $\mathcal{E}_{0}$ is the initial error defined by

$$
\mathcal{E}_{0}=p_{0}(x)-\sum_{j=1}^{m} \alpha_{j} p_{0 \mid 0}^{(j)}(x)
$$

and the error $\varepsilon_{j-1}$ is given by

$$
\varepsilon_{j-1}=\left|\mathcal{P}_{j \mid j-1}\left(p_{j-1}(x)\right)-\Psi_{j \mid j-1}\left(p_{j-1}(x)\right)\right| .
$$

Proof: Note that

$$
\begin{aligned}
\mathcal{E}_{n}= & \mathcal{P}_{n \mid n-1}\left(\Pi_{k=1}^{n-1} \mathcal{P}_{k \mid k-1}\left(p_{0}(x)\right)\right)-\Psi_{n \mid n-1}\left(\Pi_{k=1}^{n-1} \mathcal{P}_{k \mid k-1}\left(p_{0}(x)\right)\right) \\
& +\Psi_{n \mid n-1}\left(\Pi_{k=1}^{n-1} \mathcal{P}_{k \mid k-1}\left(p_{0}(x)\right)\right)-\Psi_{n \mid n-1}\left(\prod_{k=1}^{n-1} \Psi_{k \mid k-1}\left(\sum_{j=1}^{m} \alpha_{j} p_{0 \mid 0}(x)\right)\right) .
\end{aligned}
$$


Thus we have

$$
\left|\mathcal{E}_{n}\right| \leq \gamma\left|\mathcal{E}_{n-1}\right|+\varepsilon_{n-1}
$$

which leads to $(7.9)$.

Remark 7.2: Suppose $p_{0}(x)$ is a mixed Gaussian distribution and we set $p_{0 \mid 0}(x)=p_{0}(x)$. Also assume that $f(x)$ and $h(x)$ are affine functions. Then we can show that $\left|\mathcal{E}_{n}\right|=0$, i.e., the mixed Gaussian filter is exact, since the Gaussian filter is exact for the Gaussian distribution in this case.

To understand the validatity and usage of error formula (7.9) we adopt the following filter (which is different from the one we implemented), i.e., the mixed Gaussian filter with fixed means and covariances. For simplicity of our discussion we assume $n=1$. Let us consider the fixed Gaussian elements:

$$
\phi_{j}(x)=\frac{1}{\sqrt{2 \pi \sigma}} e^{-\frac{\left(x-x_{j}\right)^{2}}{2 \sigma}}, 1 \leq j \leq m
$$

with nodal points $x_{j} \in R$ and covariance $\sigma>0$.

Then we define the subspace $V^{m}$ of the probability distributions on $R$ by

$$
V^{m}=\operatorname{span}\left\{\phi_{j}\right\}_{j=1}^{m} .
$$

Define $P^{m}$ is the projection of $L^{2}(R)$ onto $V^{m}$.

We consider the mixed Gaussian filter $\hat{\Psi}_{k \mid k-1}$ with fixed means and covariances, i.e.,

$$
p_{k}(x)=P^{m}\left(\Psi_{k \mid k-1} p_{k-1}\right)=\hat{\Psi}_{k \mid k-1}\left(p_{k-1}\right), \quad p_{k-1} \in V^{m}
$$

where

$$
p_{k}(x)=\sum_{j=1}^{m} \beta_{j} \phi_{j}(x) \in V^{m} .
$$

Thus $\hat{\Psi}_{k \mid k-1}$ is the fixed operator in this case. Hence $\varepsilon_{j}, j \geq 0$ can be estimated based on the error estimate formula (7.9), assuming the smoothness of $p_{j-1}(x)$.

\section{Numerical Results and Comparisons}

In this section we demonstrate the feasibility of our proposed filter algorithms, Gauss-Hermite filter (GHF) and central difference filter ( CDF), using the three test examples as in Examples 8.1-8.3. We compare the performance of our proposed filters against that of the extended Kalman filter (EKF) and the filter of Julier-Uhlmann (JUF) [12].

We use the average root mean square error for our comparison of the methods. The average root mean square error is defined by

$$
\epsilon_{j}(k)=\sqrt{\frac{1}{N} \sum_{i=1}^{N}\left[x_{j}^{i}(k)-\hat{x}_{j}^{i}(k)\right]^{2}}
$$


which is based on $N$ different simulation runs. Here subscript $j$ denotes the $j$-th component of the vector $x(k)$ and its corresponding estimate $\hat{x}(k)$, and the superscript $i$ denotes the $i$-th simulation run.

Note that for one dimensional system the filter of Julier-Uhlmann [12] coincides with the 3 -point Gauss-Hermite filter when $\kappa=2$. Also when the stepsize is $\sqrt{3}$, the central difference filter has the same estimate algorithm as the filter of Julier-Uhlmann except for the estimation $P_{x z}$. Hence, in this section the stepsize is always chosen as $\sqrt{3}$ for our study.

We also note that our proposed filter algorithms maintain the non-negative definiteness of the covariance update.

We carried out our comparison study using various examples and different starting states $x_{0 \mid 0}$ and different problem data, but we only present the selected results in what follows.

Example 8.1 We consider the one-dimensional signal process

$$
x(k)=x(k-1)+\Delta t f(x(k-1))+w(k)
$$

and the observation process

$$
y(k)=\Delta t h(x(k))+v(k)
$$

where

$$
f(x)=5 x\left(1-x^{2}\right), \quad h(x)=(x-0.05)^{2}
$$

and

$$
w(k) \sim N\left(0, b^{2} \Delta t\right) \quad v(k) \sim N\left(0, d^{2} \Delta t\right)
$$

It is motivated from the continuous time systems (6.1) and (6.2) of the form

$$
\begin{aligned}
& d x(t)=f(x) d t+b d w(t) \\
& d y(t)=h(x) d t+d d v(t)
\end{aligned}
$$

as described in Section 6 .

This example is used as a benchmark because of the following reasons. The deterministic equation

$$
\dot{x}=f(x)
$$

has two stable equilibria -1 and 1 , and one unstable equilibrium 0 . So, we observed that the signal process $x_{k}$ is distributed around one of stable equilibria. The observation function $h(x)=(x-0.05)^{2}$ is the square of shifted distance from the origin and distinguishs the two stable equilibria marginally. The performance of the filter is possibly tested because of the minor margin of detecting measurement function $h(x)$ of the signal process.

We use the following problem data: the time elapse $\Delta t=0.01$; the initial condition $x_{0}=-0.2$, i.e., $x(0)=-0.2$; the starting point $\hat{x}_{0 \mid 0}=0.8, P_{0 \mid 0}=P_{0}=2$; the system parameters $b=0.5$ and $d=0.1$. We consider the time from 0 to 4 . $\kappa$ is chosen as the best value, i.e., $\kappa=2$ (see [12]), 


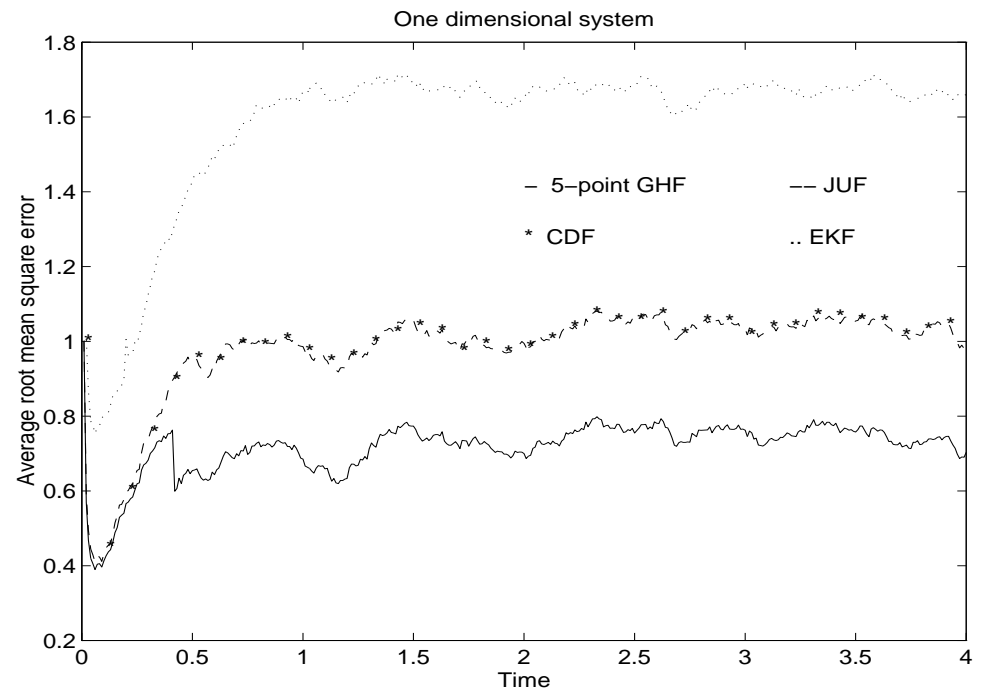

Figure 1: The Average of Root Mean Square Error

Figure 1 shows the average root mean square errors committed by each filter across a simulation consisting of 50 runs. From Figure 1 we see that the 5-point Gauss-Hermite filter performs better than others. The central difference filter has similar performance like the filter of Julier-Uhlmann [12] in this example. All the three filters perform superior to the extended Kalman filter.

Example 8.2 In this example, we consider the Lorenz system

$$
x(k)=x(k-1)+\Delta t f(x(k-1))+b w(k), \quad y(k)=\Delta t h(x(k))+d v(k)
$$

where $x=\left[x_{1}, x_{2}, x_{3}\right]^{t}$ is the $R^{3}$-valued process. The $R$-valued processes $w(k)$ and $v(k)$ are white noises with the same covariance $\Delta t$. Here $b \in R^{3}$ is a constant vector and $d \in R$ is a constant scalar. Also

$$
f(x)=\left[r_{1}\left(-x_{1}+x_{2}\right), r_{2} x_{1}-x_{2}-x_{1} x_{3},-r_{3} x_{3}+x_{1} x_{2}\right]^{t}
$$

The observation function is chosen as the shifted distance from the origin given by

$$
h=\sqrt{\left(x_{1}-0.5\right)^{2}+x_{2}^{2}+x_{3}^{2}} .
$$

It is motivated from the Lorenz stochastic differential system of the form (8.4) as described in Section 6 where $x \in R^{3}$.

The three dimensional deterministic equations

$$
\dot{x}=f(x)
$$




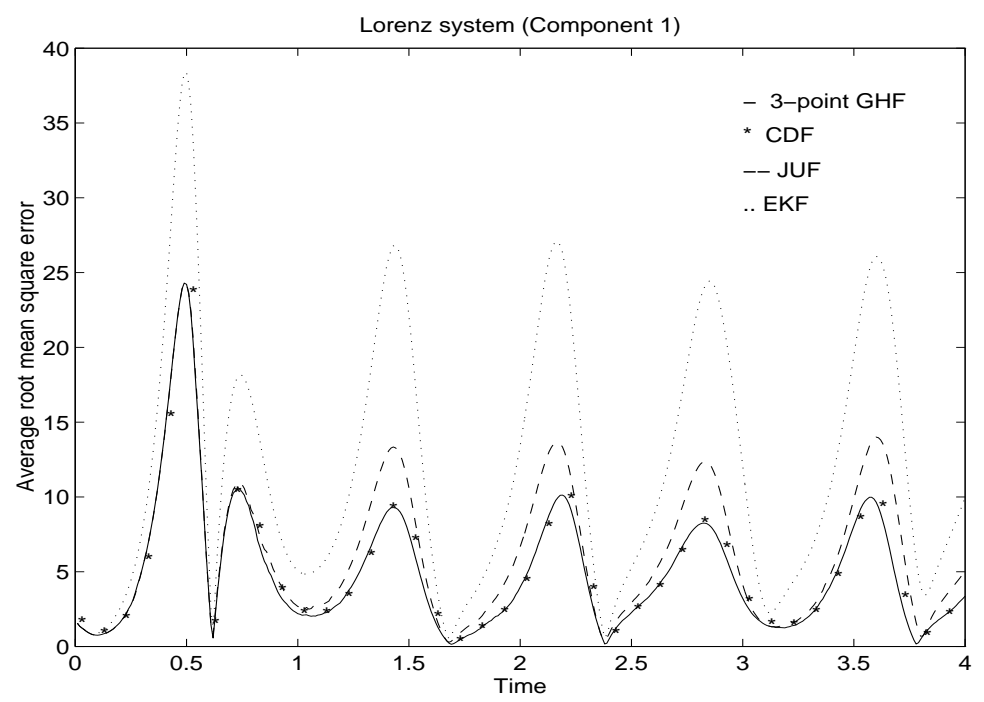

Figure 2: The Average of Root Mean Square Error

is the Lorenz equations in which $r_{j}(j=1,2,3)$ are positive parameters. The three parameters have a great deal of impact on the system. Here, we choose $r_{1}=10, r_{2}=28$, and $r_{3}=8 / 3$ which is a mathematically very interesting case, because in the case there are three unstable equilibria and a strange attractor for the equations.

We use the following problem data: the system parameters are chosen as $b=[0,0,0.5]^{t}$ and $d=0.065$; the initial condition is $x_{0}=[-0.2,-0.3,-0.5]^{t}$, i.e., $x(0)=[-0.2,-0.3,-0.5]^{t}$, and $\Delta t=0.01$. We consider the time from 0 to 4 . The initial estimate is $\hat{x}_{0 \mid 0}=[1.35,-3,6]^{t}$ with covariance $P_{0 \mid 0}=0.35 I$.

In Figures 2-4 we show the average of root mean square errors for each component of system state committed by the algorithms of the Gauss-Hermite quadrature rule, the central difference approximation and the JU-rule respectively across a simulation consisting of 50 runs. As shown in Figures 2-4, the GHF and the CDF have smaller errors than the JUF and the EKF. From these figures we conclude that in this example the GHF has a substantial improved performance, and the GHF performs a little better than the CDF.

Example 8.3 We further discuss the three-dimensional continuous signal process

$$
\dot{x}(t)=f(x(t))+w(t)
$$

and the discrete observation process

$$
y(t)=\sqrt{\left(\tilde{M}^{2}+\left[x_{1}(t)-H\right]^{2}\right)}+r(t)
$$

where $w(t) \in R^{3}$ and $r(t) \in R$ are zero mean and uncorrelated noises with covariances given by $Q(t) \in R^{3 \times 3}$ and $R(t) \in R$, respectively. The function $f(x)$ is given by

$$
f(x)=\left[-x_{2},-e^{-\gamma x_{1}} x_{2}^{2} x_{3}, 0\right]^{t} .
$$




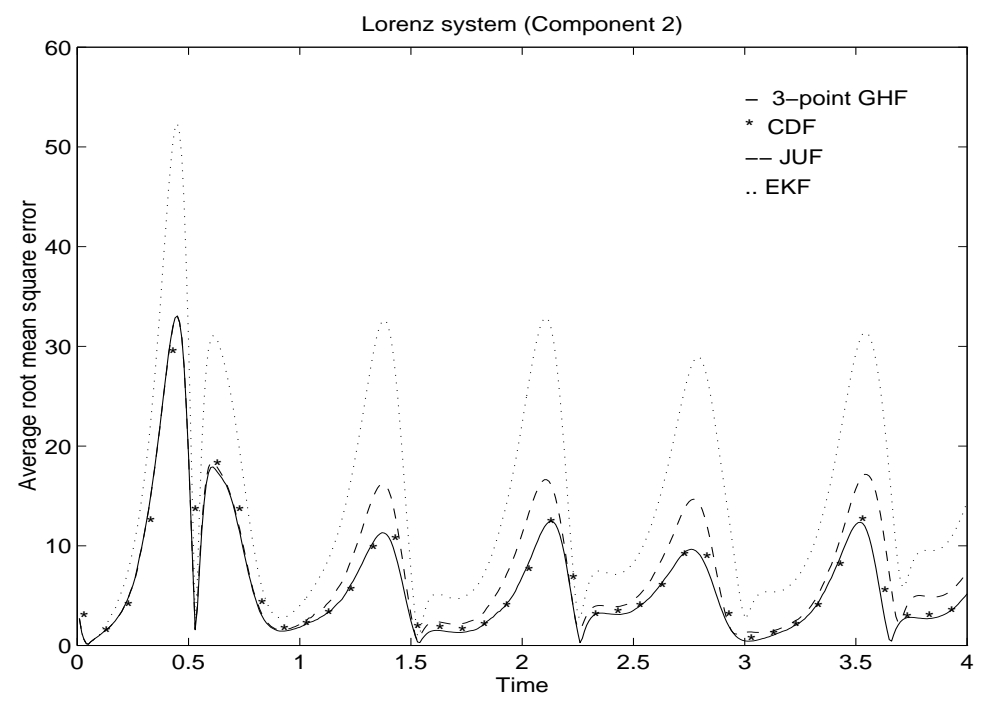

Figure 3: The Average of Root Mean Square Error

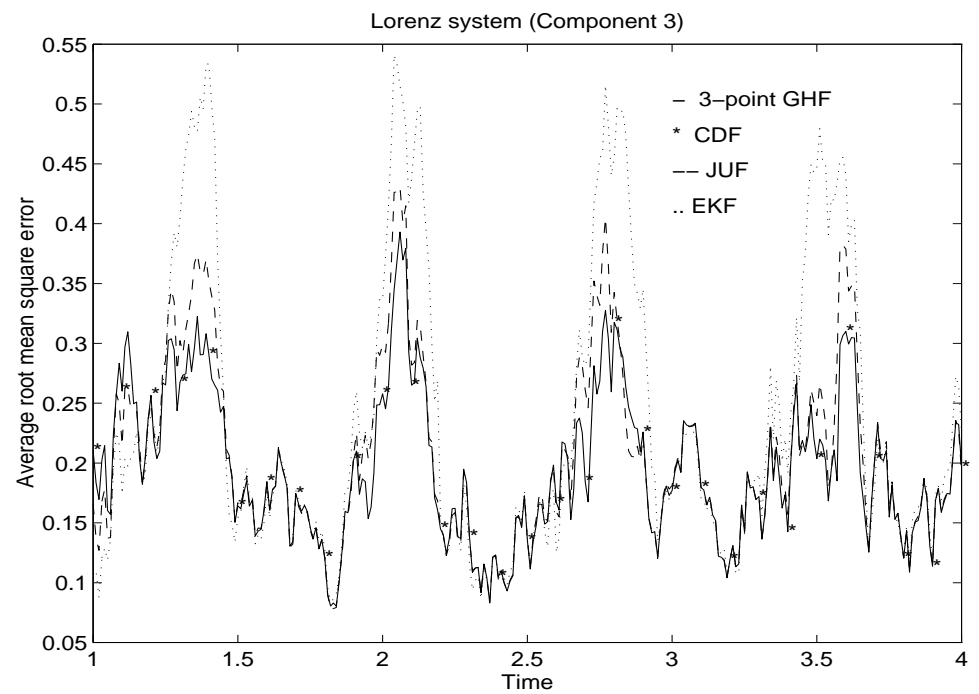

Figure 4: The Average of Root Mean Square Error 
The above system states $x_{1}, x_{2}$ and $x_{3}$ represent altitude (in feet), velocity (in feet per second) and constant ballistic coefficient (in per second), respectively. The detail physical meaning of the system and its parameters can be found in [2].

We choose this example as a benchmark because it contains significant nonlinearities in the signal and observation processes, and had been discussed widely in the literature.

In the previous literature, a fourth-order Runge-Kutta scheme with 64 steps between each observation is employed to solve numerical intergration of (8.8) based on the significant signal process $[2,12]$. That is, in the predicator steps of EKF and JUF, the means are calculated using the numerical scheme. Then their covariance are propagated from the $j$ th to $(j+1)$ th step using

$$
P_{t_{k}+(j+1) \delta \mid t_{k}}=\psi\left(t_{k}+(j+1) \delta, t_{k}+j \delta\right) P_{t_{k}+j \delta} \psi^{t}\left(t_{k}+(j+1) \delta, t_{k}+j \delta\right)
$$

with

$$
\psi\left(t_{k}+(j+1) \delta, t_{k}+j \delta\right) \simeq I+\delta J_{f}+\frac{1}{2} \delta^{2} J_{f}^{2}
$$

where $J_{f}$ was evaluated at $t_{k}+n \delta$ and $\delta=\Delta t / 64$. Thus, $P_{k+1 \mid k}=P_{t_{k}+64 \delta \mid t_{k}}$.

In the comparison, we implement the proposed approximation schemes $(6.7)-(6.9)$ to solve the filtering problem of (8.8)-(8.9). In the predictor step of GHF, we directly use the Euler approximation of signal process (8.8) based on 32 or 64 steps (i.e., $M=32$ or 64 ) between each observation (they are shortly denoted by GHF32 or GHF64, respectively).

However, in NGHF the signal process system (8.8) is rewritten as the discrete form

$$
x_{k}^{j}=x_{k}^{j-1}+\delta F\left(x_{k}^{j-1}\right)+\sqrt{\delta} w
$$

where $x_{k}^{j}$ is the approximation of the process $x(t)$ at $t=k \Delta t+j \delta$, and function $F(s)=$ $\frac{1}{6}\left(k_{1}+2 k_{2}+2 k_{3}+k_{4}\right)$ with

$$
\begin{aligned}
& k_{1}=f(s, \delta), k_{2}=f\left(s+0.5 \delta k_{1}, \delta\right), \\
& k_{3}=f\left(s+0.5 \delta k_{2}, \delta\right), k_{4}=f\left(s+\delta k_{3}, \delta\right),
\end{aligned}
$$

which is the fourth-order Runge-Kutta scheme. Especially, $\delta=\Delta t / 64$ in NGHF64.

We use the following data: the system parameters are chosen as $\gamma=5 \times 10^{-5}, H=10^{5}$, $\tilde{M}=10^{5}, Q(k)=0$ and $R(k)=10^{4}$; the initial condition is $x(0)=\left[3 \times 10^{5}, 2 \times 10^{-4}, 10^{-3}\right]^{t}$ and $\Delta t=0.1$. We consider the time from 0 to 30 . The initial estimate is $\hat{x}_{0 \mid 0}=\left[3 \times 10^{5}, 2 \times\right.$ $\left.10^{-4}, 3 \times 10^{-5}\right]^{t}$ with covariance

$$
P_{0 \mid 0}=\left[\begin{array}{ccc}
10^{6} & 0 & 0 \\
0 & 4 \times 10^{6} & 0 \\
0 & 0 & 10^{-4}
\end{array}\right] .
$$

We also choose the optimization number $\kappa=0$ in JUF for the three dimensional system.

In Figures 5-7 we show the absolute value of average error for each component committed by the algorithms of the Gauss-Hermite quadrature rule, the JU-rule and the EKF respectively across a simulation consisting of 50 runs. These figures show that in this example the GHF has superior performance than the JUF and the EKF. 


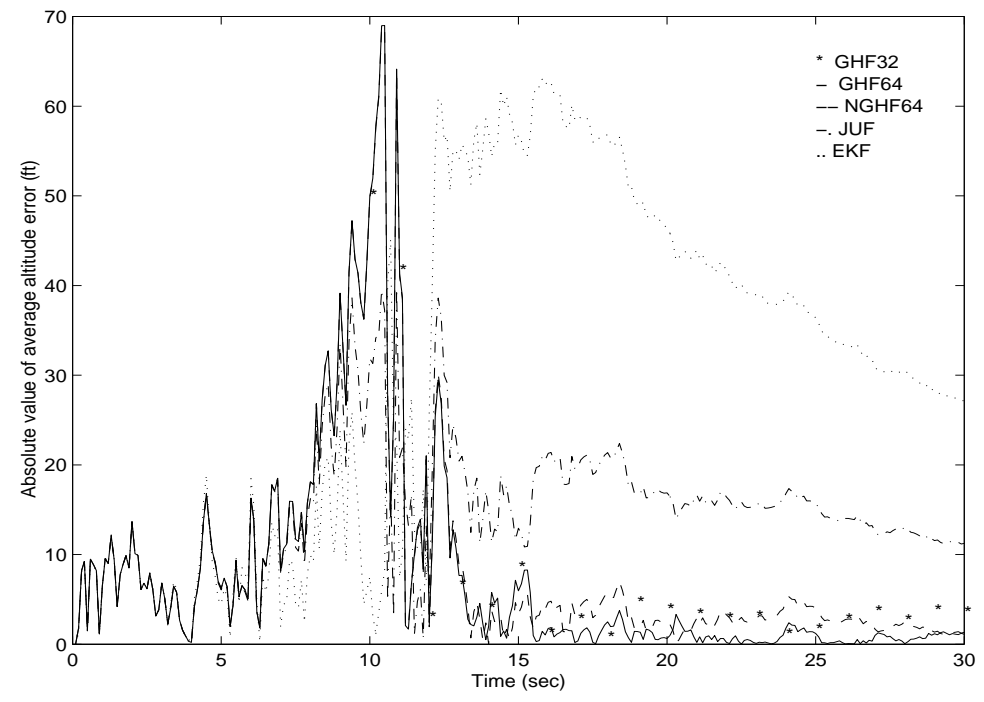

Figure 5: The Absolute Value of Average Altitude Error

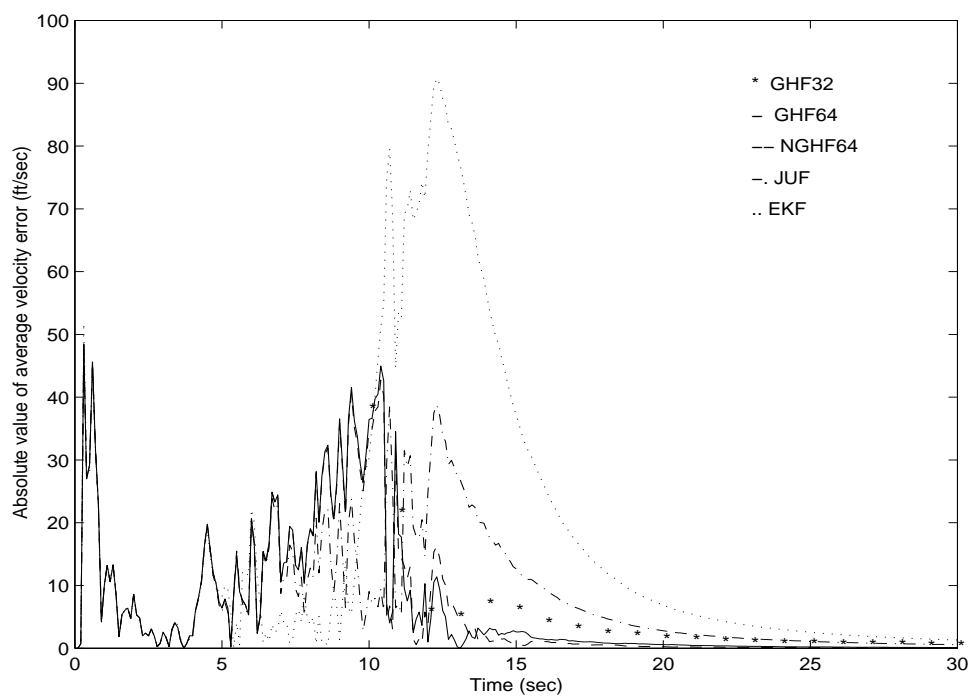

Figure 6: The Absolute Value of Average Velocity Error 


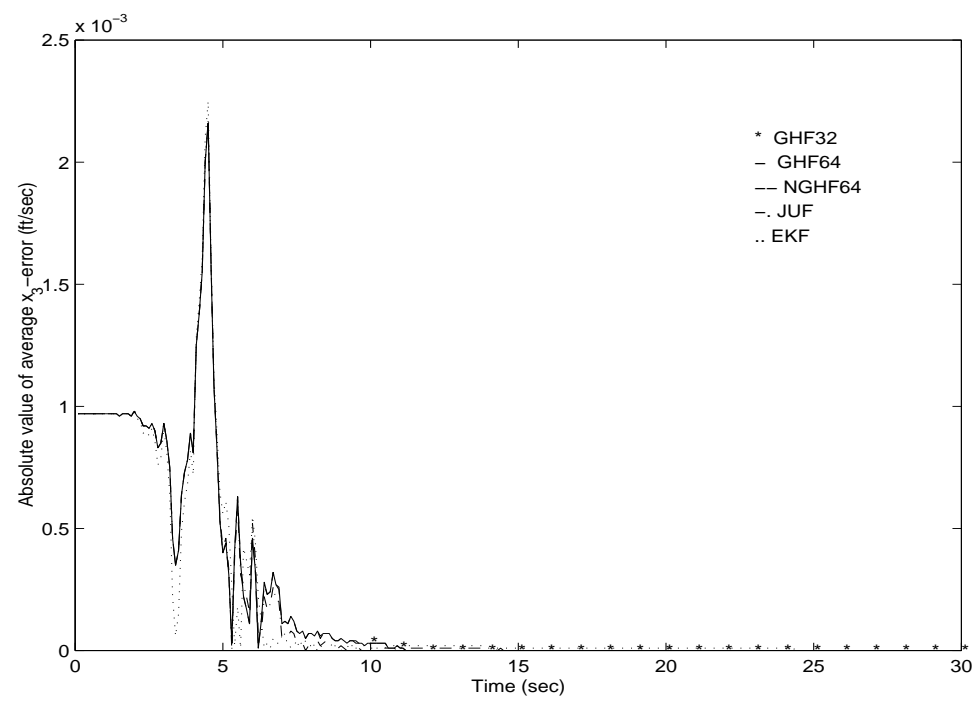

Figure 7: The Absolute Value of Average $x_{3}$-Error

\subsection{Mixed Gaussian Example}

In this section we first demonstrate a nearly optimal performance of the mixed Gaussian method described in Section 5. We consider the same example as in Example 8.1 with $h(x)=|x-.5|$. We employ the two Gaussian distributions starting from

$$
x_{0 \mid 0}^{(1)}=.5, \quad P_{0 \mid 0}^{(1)}=.1 \quad \text { and } \quad x_{0 \mid 0}^{(2)}=-.5, \quad P_{0 \mid 0}^{(2)}=.1,
$$

respectively and the initial weights are $\alpha_{0}^{(1)}=\alpha_{0}^{(2)}=.5$. In order to demonstrate the effectiveness of the proposed update (5.5)-(5.6) we jumped the process $x_{k}$ at $k=150$ (which corresponds to $t=1.5$ in the continuous time process (8.4)) to $x_{150}=.5$. It may represent an impulse force at $t=1.5$. We compare the performance of the mixed Gaussian filter against the one of the optimal Zakai filter (6.4) with $\Delta t=.01$ in Figures 8-11. Here GHF1 (GHF2) represents the 3-point Gauss-Hermite filter which is the first (second) component of the two mixed Gaussian filter with Weight 1 (Weight 2). We approximate the Zakai equation by the operator-splitting method as described in [10]. We observed that the mixed Gaussian filter performs nearly optimally up to $t=1.5$. We also note that the Zakai filter is no longer optimal after $k=150$ because of the jump. As seen as Figures 8-9, the update formula (5.5)-(5.6) quickly captures the phase change from the one steady point $(x=-1)$ to the other $(x=1)$. The observations are also supported by the comparison in Figures 10-11.

Example 8.3 (Revisted) Next, we also apply our mixed Gaussian method to Example 8.3. We employ the two Gaussian distribution starting from

$$
x_{0 \mid 0}^{(1)}=\left[3 \times 10^{5}, 2 \times 10^{-4}, 3 \times 10^{-5}\right]^{t} \quad \text { and } \quad x_{0 \mid 0}^{(2)}=\left[3 \times 10^{5}, 2 \times 10^{-4}, 10^{-1}\right]^{t}
$$




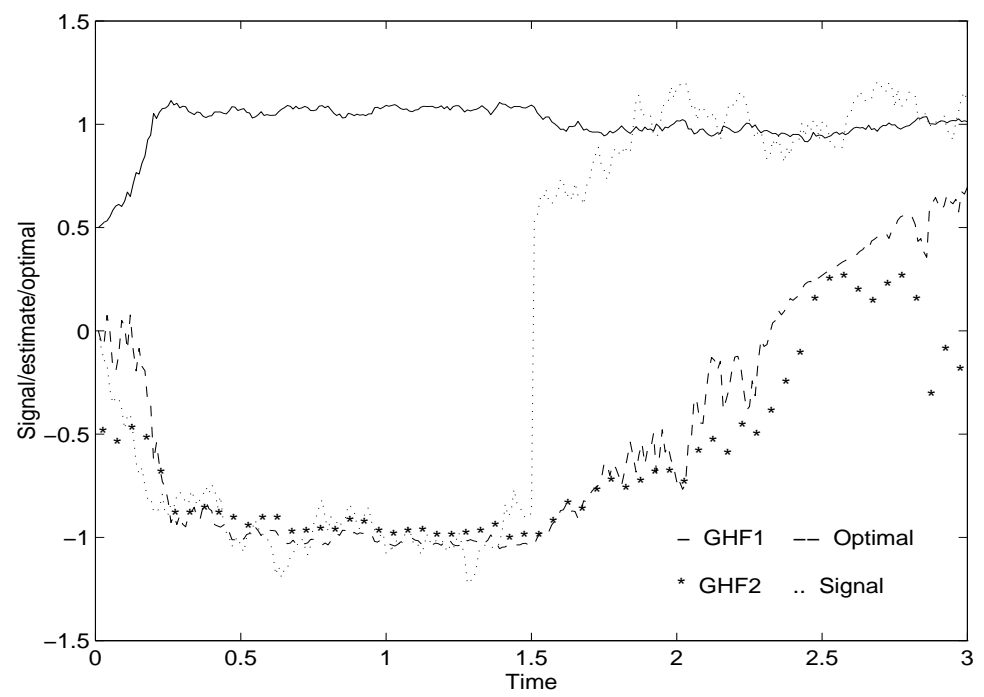

Figure 8: The comparison of signal, estimates of two mixed Gaussian filter and optimal filter

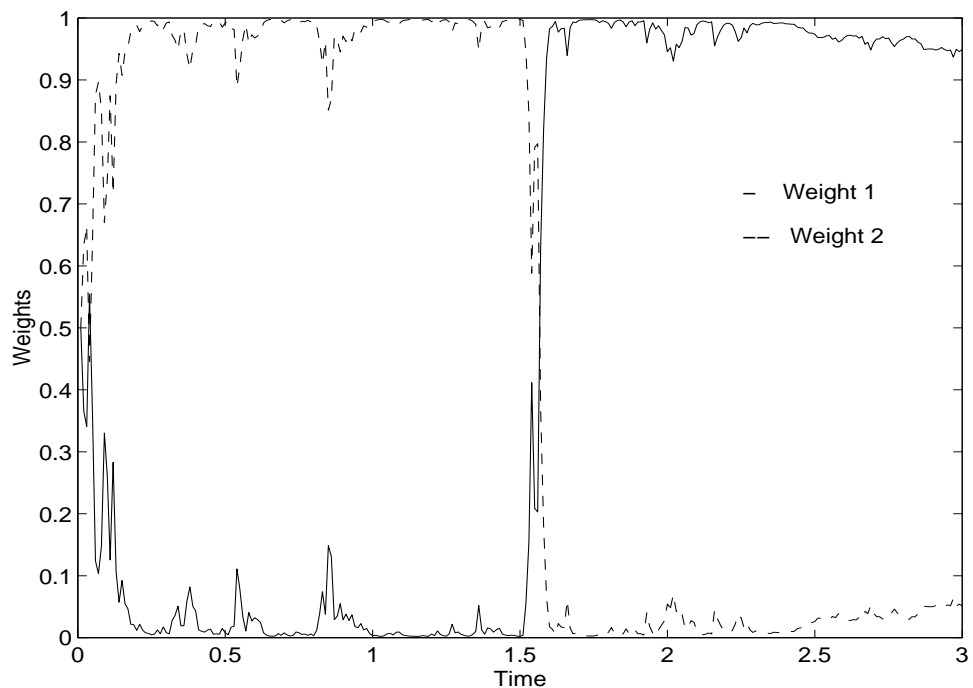

Figure 9: The weights of two mixed Gaussian filter 


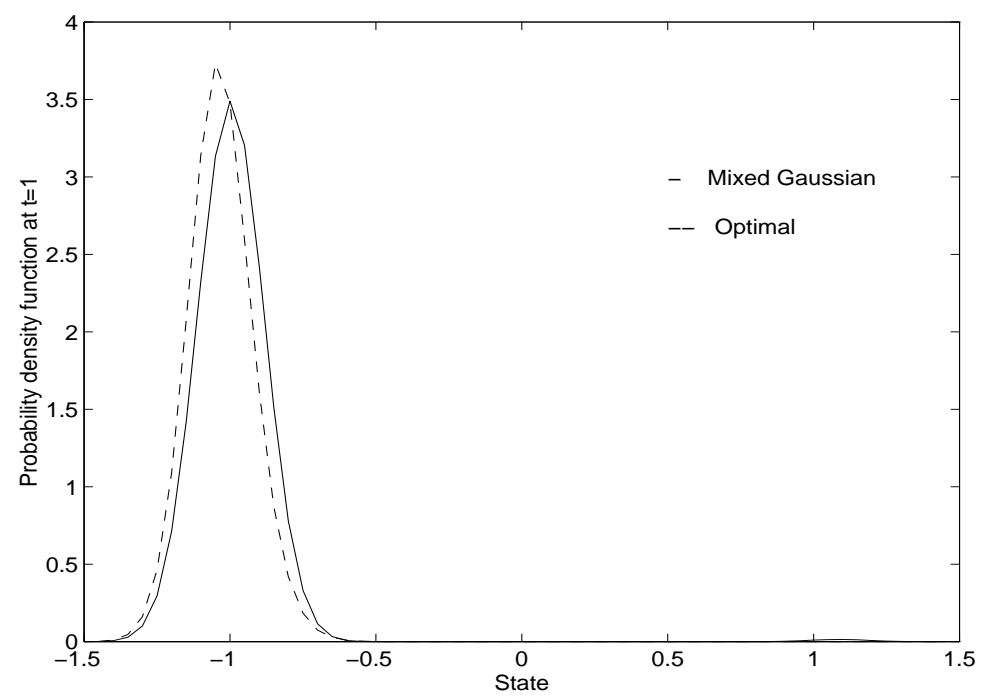

Figure 10: The comparison of the probability density functions of mixed Gaussian filter and optimal filter at $t=1$

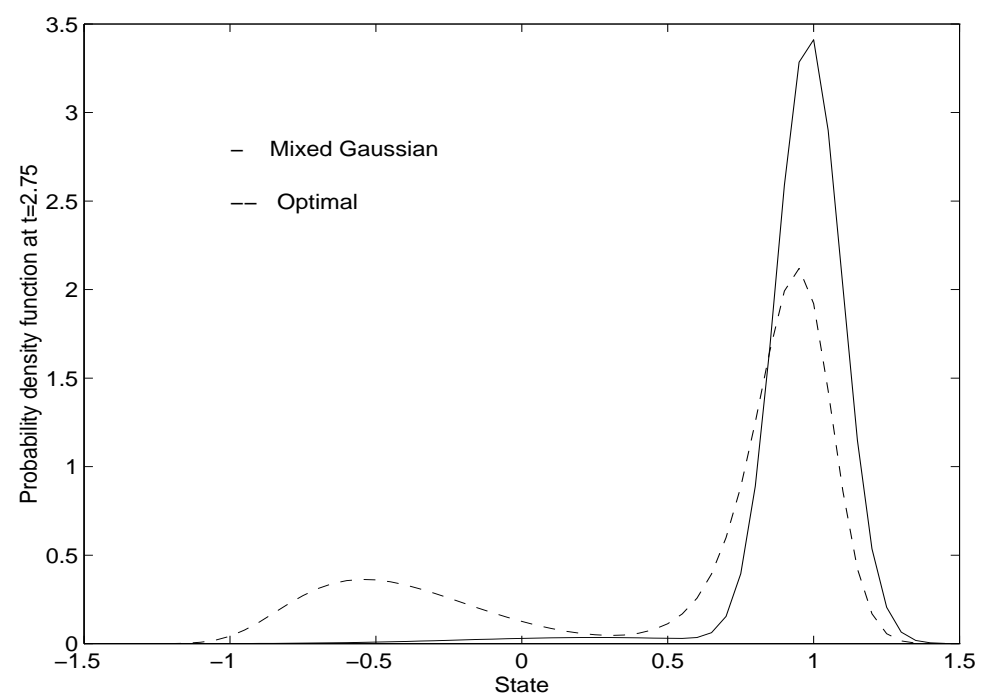

Figure 11: The comparison of the probability density functions of mixed Gaussian filter and optimal filter at $t=2.75$ 
with the same covariance

$$
P_{0 \mid 0}=\left[\begin{array}{ccc}
10^{6} & 0 & 0 \\
0 & 4 \times 10^{6} & 0 \\
0 & 0 & 10^{-4}
\end{array}\right] \text {. }
$$

respectively and the initial weights are $\alpha_{0}^{(1)}=\alpha_{0}^{(2)}=\frac{1}{2}$. We demonstrate the performance of the mixed Gaussian filter with $\Delta t=0.1$ in Figures 12-15. We see that the mixed Gaussian filter based on the update formula (5.5)-(5.6) captures the signal process very well. That is, the weights of the two mixed Gaussian filters shown in Figure 15 effectively pick up the combined performance of the Gaussian filters: GHF1 and GHF2. More detailed discussions and numerical testings of the mixed Gaussian filter will be presented in the forthcoming paper.

\section{Conclusions}

The paper presents the systematic formulation of Gaussian filters and mixed Gaussian filters based on the efficient numerical intergation of the Bayesian formula for optimal recursive filter. Based on our formulation we develop the two filter algorithms, namely, the GaussHermite filter (GHF) and the central difference filter (CDF). We demonstrated the feasibility of our proposed filter algorithms for testing nonlinear filtering problems. Our numerical results indicate that both the Gauss-Hermite filter and the central difference filter have superior performance to the filter of Julier-Uhlmann and the extended Kalman filter. We also proposed the new update rules for the Gauss sum filters and show that they can perform near optimally.

\section{Acknowledgement}

We thank the referees for their careful reviews and helpful suggestions. 


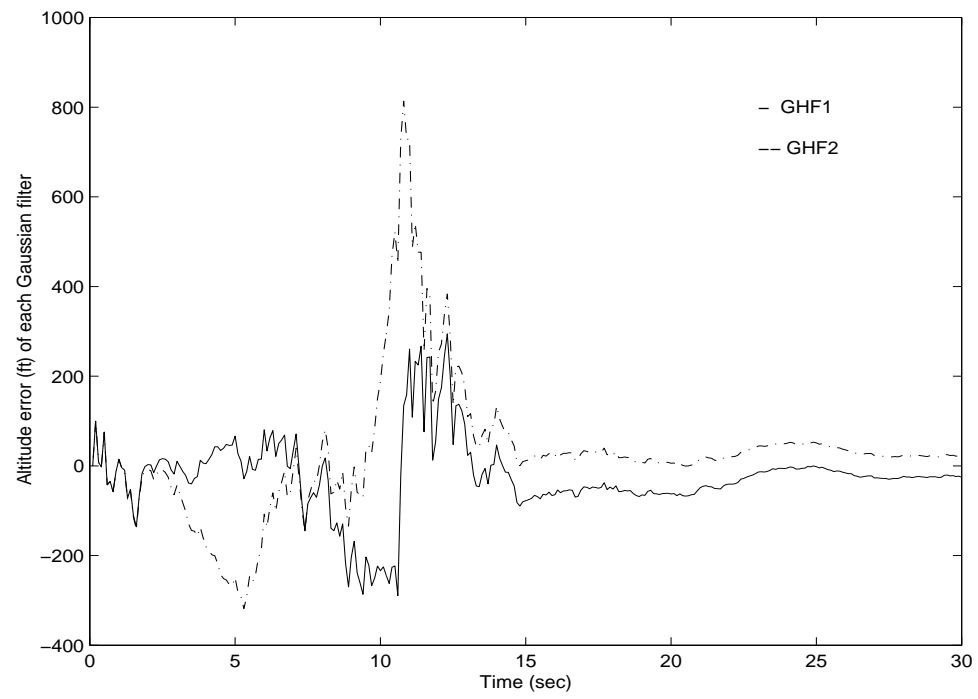

Figure 12: The comparison of altitude errors of each Gaussian filter

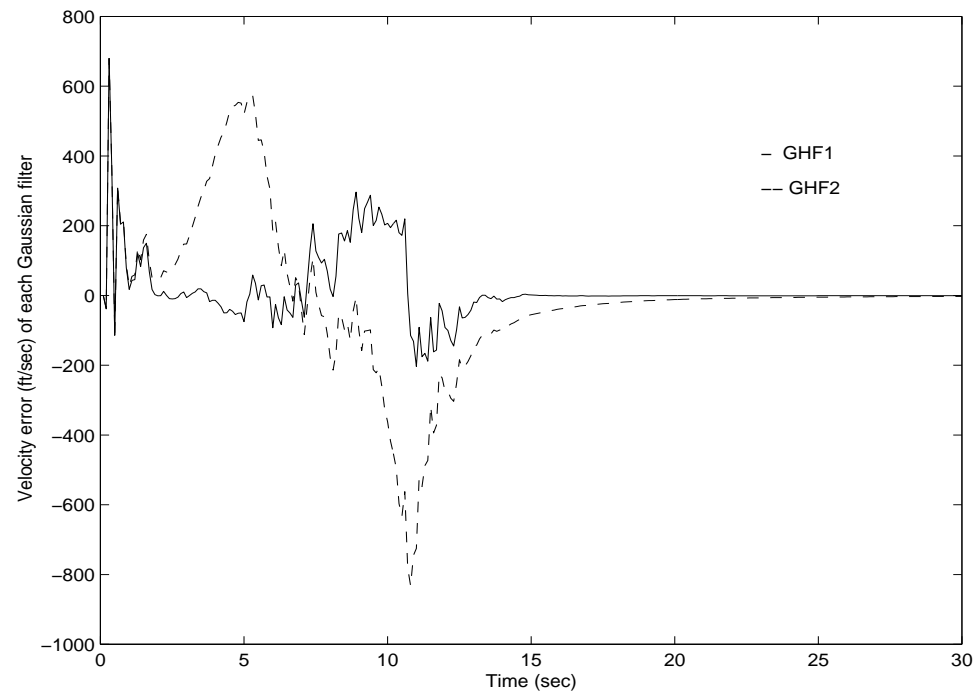

Figure 13: The comparison of velocity errors of each Gaussian filter 


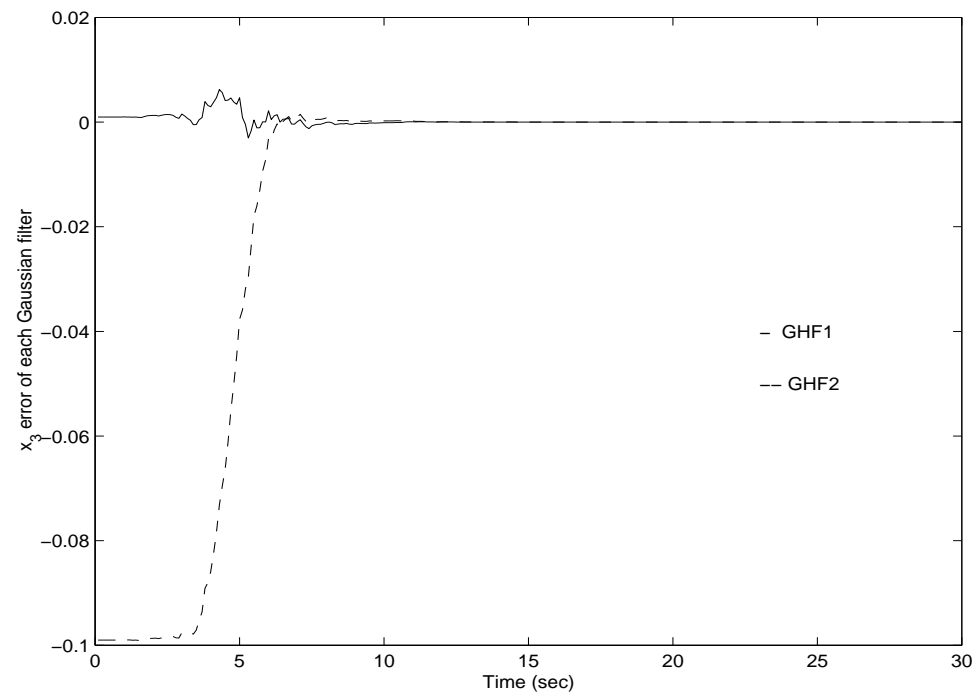

Figure 14: The comparison of $x_{3}$ errors of each Gaussian filter

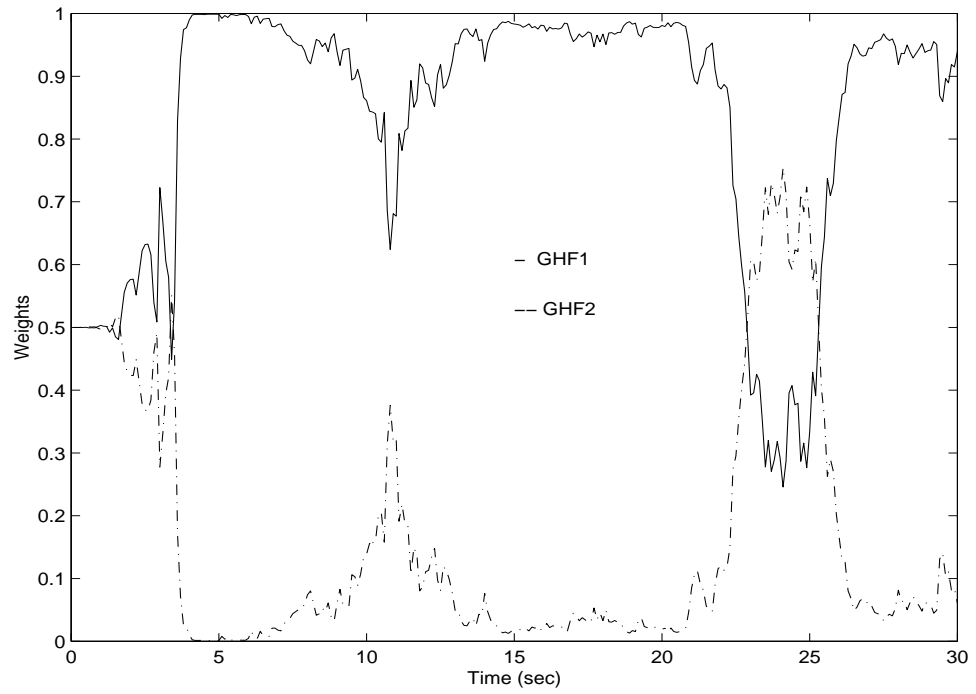

Figure 15: The weights of two mixed Gaussian filters 


\section{References}

[1] D. L. Alspace and H. W. Sorenson, "Nonlinear Bayesian estimation using Gaussian sum approximation," IEEE Trans. Autom. Control, vol. 17, no. 4, pp. 439-448, 1972.

[2] M. Athans, R. P. Wishner and A. Bertolini, "Suboptimal state estimation for continuous-time nonlinear systems from discrete noise measurements," IEEE Trans. Autom. Control, vol. 13, no. 5, pp. 504-514, 1968.

[3] A. Bensoussan, "Nonlinear filtering theory," in Progress in Automation and Information Systems, Recent Advances in Stochastic Calculus, J. S. Baras and V. Mirelli, eds, Springer-Verlag, New York, 1990.

[4] A. Bensoussan, R. Glowinski, and A. Rascanu, "Approximation of the Zakai equation by the splitting up method," SIAM J. Control Optim., vol 28, pp. 1420-1431, 1990.

[5] Y. Bar-Shalom and X. R. Li, "Estimation and tracking: principles, techniques, and software," Artech House, 1993.

[6] J. E. Dennis and R. B. Schnabel, "Numerical methods for unconstrained optimization and nonlinear equations," Prentice-Hall, Inc, Englewood Cliffs, New Jersey, 1983.

[7] C. P. Fang, "New algorithms of Gaussian assumed density filter and a convergence result," submitted to IEEE Trans. Autom. Control.

[8] P. Florchinger and F. Le Gland, "Time-discretization of the Zakai equation for diffusion process observed in correlated noise," in 9th Conference on Analysis and Optimization of Systems, Lecture Notes on in Control and Inform Sci., 144, A. Bensoussan and J. L. Lions, eds, Springer-Verlag, New York, 1990.

[9] G. H. Golub, "Some modified matrix eigenvalue problems," SIAM Rev., vol. 15, pp. 318-334, 1973.

[10] K. Ito, "Approximation of the Zakai equation for nonlinear filtering," SIAM J. Control Optim., vol 34, pp. 620-634, 1996.

[11] A. H. Jazwinski, Stochastic process and filtering theory, Academic Press, New York and London, 1970.

[12] S. J. Julier and J. K. Uhlmann, A General method for approximating nonlinear transformations of probability distributions, WWW http://phoebe. robots.ox.ac.uk/default.htm, 8(1994).

[13] S. J. Julier, J. K. Uhlmann and H. F. Durrant-Whyte, "A new approach for filtering nonlinear systems," Proceedings of the American Control Conference, Seattle, Washington, pp. 1628-1632, June, 1995. 
[14] R. E. Kalman, "A new approach to linear filtering and prediction problems," Trans. ASME, J. Basic Eng., vol. 82D, pp. 35-45, Mar. 1960.

[15] H. J. Kushner, "Approximations to optimal nonlinear filters," IEEE Trans. Autom. Control, vol. 12, no. 5, pp. 546-556, 1967.

[16] H. J. Kushner, private communication.

[17] V. Mazya and G. Schmidt, "On approximate approximations using Gaussian kernels," IMA J. Numer. Anal., vol. 16, pp. 13-29, 1996.

[18] M. Piccioni, "Convergence of implicit discretization schemes for linear differential equations eith application to filtering," Lecture Note in Mathematics, 1236, Stochastic Partial Differential Equations and Application, Trento, 1985, ed. G. Da Prato and L. Tabbaro.

[19] B. L. Rozovskii, "Stochastic evolution systems, linear theory and application to nonlinear filtering," Math. Appl., Kluwer Academic Publishers, Norwell, MA, 1991.

[20] H. W. Sorenson and D. L. Alspace, "Recursive Bayesian estimation using Gaussian sums," Automatica, vol. 7, pp. 465-479, 1967. 